\title{
A Computationally Efficient Algorithm for Determining Regional Cerebral Blood Flow in Heterogeneous Tissues by Positron Emission Tomography
}

\author{
Kathleen Schmidt* and Louis Sokoloff
}

\begin{abstract}
Inclusion of brain tissues with different rates of blood flow and metabolism within a voxel or region of interest is an unavoidable problem with positron emission tomography due to its limited spatial resolution. Because regional cerebral blood flow (rCBF) is higher in gray matter than in white matter, the partial volume effect leads to underestimation of $\mathrm{rCBF}$ in gray matter when $\mathrm{rCBF}$ in the region as a whole is determined. Furthermore, weighted-average $\mathrm{rCBF}$ itself is underestimated if the kinetic model used in the analysis fails to account for the tissue heterogeneity. We have derived a computationally efficient method for estimating both gray matter and weighted-average rCBF in heterogeneous tissues and validated the method in simulation studies. The method is based on a model that represents a heterogeneous tissue as a weighted mixture of two homogeneous tissues. A linear least squares algorithm is used to estimate the model parameters.
\end{abstract}

Index Terms-Brain, blood flow, least squares methods, positron emission tomography.

\section{INTRODUCTION}

$\mathbf{M}$ ETHODS for determination of regional cerebral blood flow ( $\mathrm{rCBF}$ ) with freely diffusible tracers are based on the equation that Kety derived in his analyses of the principles of inert gas exchange [1], namely

$$
\frac{d C_{i}}{d t}=-\left(\frac{F}{\lambda}\right) C_{i}(t)+F C_{a}(t)
$$

where

$C_{i}$ and $C_{a}$ concentrations of tracer in tissue and arterial blood, respectively;

$F \quad$ rate of blood flow per unit mass of tissue, and

$\lambda$ tissue:blood partition coefficient for the tracer and tissue in question.

Equation (1) assumes tissue homogeneity and diffusion equilibrium between blood and tissue. For methods used in the satura-

\footnotetext{
Manuscript received September 25, 2000; revised April 20, 2001. The Associate Editor responsible for coordinating the review of this paper and recommending its publication was $\mathrm{C}$. Thompson. Asterisk indicates corresponding author.

*K. Schmidt is with the Laboratory of Cerebral Metabolism, National Institute of Mental Health, Building 36, Room 1A-07, 9000 Rockville Pike, Bethesda, MD 20892-4030 USA (e-mail: kathy@ shiloh.nimh.nih.gov).

L. Sokoloff is with the Laboratory of Cerebral Metabolism, National Institute of Mental Health, Bethesda, MD 20892-4030 USA.

Publisher Item Identifier S 0278-0062(01)05361-7.
}

tion mode, $t$ is the time after injection of tracer into the blood and $C_{i}(0)=C_{a}(0)=0$. Algorithms for determining cerebral blood flow are based on the solution of (1), i.e.,

$$
C_{i}(T)=F \int_{0}^{T} C_{a}(t) e^{-(F / \lambda)(T-t)} d t
$$

or an integration of (1), i.e.,

$$
C_{i}(T)=-\left(\frac{F}{\lambda}\right) \int_{0}^{T} C_{i}(t) d t+F \int_{0}^{T} C_{a}(t) d t
$$

The first method for measurement of $\mathrm{rCBF}$ in man with positron emission tomography (PET) used $\mathrm{H}_{2}^{15} \mathrm{O}$ as the tracer [2], [3]. The $\mathrm{H}_{2}^{15} \mathrm{O}$ method relied on a single integrated tissue measurement acquired after a finite interval of time following administration of the tracer, and (2), integrated over the same time interval, was solved for $F$ with a prespecified value for $\lambda$ [2], [4]. With advances in scanner and computer technology that allowed rapid acquisition and storage of multiple frames of data, methods were introduced that estimated $F$ and $\lambda$ simultaneously from a series of measurements of tissue concentration [5]-[12]. Several of these methods were designed to reduce reconstruction time by using integrals [9] or weighted integrals [5]-[8], [12] of the projection data, together with linear estimation procedures, for determining the parameters $F$ and $\lambda$. Linear methods, however, have been shown to possess small to moderate degrees of bias in the estimates [13]. Feng et al. [14] introduced a generalized linear least squares approach to remove the bias in the estimates arising from statistical dependencies in the data.

It was recognized from the outset that a model designed for application in homogeneous tissues might be inaccurate when applied to tissues measured with PET as the limited spatial resolution of the scanner assures that most tissue measurements represent a mixture of gray and white matter. Errors due to heterogeneity were considered to be small [2], [4], [7], [10]-[12], [15], [16]; their magnitude depends on a number of factors such as the duration of experimental period, time course of arterial concentration of tracer, values of $\lambda$, differences in rates of blood flow in the components of the mixed tissue, relative masses of tissues, and method for estimation of the parameters [17]. In simulations of PET $\mathrm{H}_{2}^{15} \mathrm{O}$ studies, weighted-average flow underestimation ranged from $<5 \%$ in short-duration studies [2], [10] to 
approximately $20 \%$ in longer-duration studies [10]. These simulations did not take into account noise in the measurements of tissue activity that has the potential to further degrade estimates of weighted-average blood flow.

The unavoidable inclusion of white matter in measurements of gray matter activity leads to a second problem. Weighted-average flow in a gray matter region depends on the amount of white matter included in the region of measurement whereas one would like to know the flow in the gray matter only. In fact, the average gray:white rCBF ratio is often considered to be indicative of the severity of the partial volume effects of the scanner. In studies that utilized the relatively high spatial resolution of quantitative autoradiography, the gray:white rCBF ratio was found to be greater than 4:1 in cortical structures in the conscious cat [18] and conscious rat [19] whereas with PET measurements in normal human brain it is at best of the order of 3:1 [20]. There have been a number of methods proposed to estimate and correct for partial volume effects, most of which require use of a high resolution magnetic resonance image of the subject coregistered to the PET image. It would be useful if the gray matter flow could be determined along with the weighted-average flow from the dynamic PET scans alone.

\section{THEORY}

Consider a tissue that is comprised of a mixture of two homogeneous tissues, e.g., gray and white matter. The model for distribution of a freely diffusible tracer in each tissue is as given in (1); the concentration of tracer in the two homogeneous tissues can be described by the set of equations

$$
\begin{aligned}
& \frac{d C_{i 1}}{d t}=-\left(\frac{F_{1}}{\lambda_{1}}\right) C_{i 1}(t)+F_{1} C_{a}(t) \\
& \frac{d C_{i 2}}{d t}=-\left(\frac{F_{2}}{\lambda_{2}}\right) C_{i 2}(t)+F_{2} C_{a}(t)
\end{aligned}
$$

where

$C_{i 1}$ and $C_{i 2} \quad$ concentrations of the tracer in Tissues 1 and 2 ;

$F_{1}$ and $F_{2} \quad$ blood flow per unit mass of Tissues 1 and 2;

$\lambda_{1}$ and $\lambda_{2} \quad$ respective tissue:blood partition coefficients for the tracer and tissues in question;

$C_{a} \quad$ concentration of tracer in the arterial blood.

The concentration of tracer in the mixed tissue is the weighted sum of the concentrations in the homogeneous tissues comprising the mixture, i.e.,

$$
C_{i}(T)=w_{1} C_{i 1}(T)+w_{2} C_{i 2}(T)
$$

where $w_{1}$ and $w_{2}=1-w_{1}$ are the relative tissue weights. For this single-input single-output system, an equation can be derived to relate $C_{i}$ to integrals of $C_{i}$ and $C_{a}$ (see Appendix I). The resultant equation is

$$
\begin{aligned}
C_{i}(T)= & -\theta_{1} \int_{0}^{T} C_{i}(t) d t-\theta_{2} \int_{0}^{T} \int_{0}^{t} C_{i}(s) d s d t \\
& +\theta_{3} \int_{0}^{T} C_{a}(t) d t+\theta_{4} \int_{0}^{T} \int_{0}^{t} C_{a}(s) d s d t
\end{aligned}
$$

where the coefficients are given by

$$
\begin{aligned}
& \theta_{1}=\left(\frac{F_{1}}{\lambda_{1}}\right)+\left(\frac{F_{2}}{\lambda_{2}}\right) \quad \theta_{2}=\left(\frac{F_{1}}{\lambda_{1}}\right)\left(\frac{F_{2}}{\lambda_{2}}\right) \\
& \theta_{3}=w_{1} F_{1}+w_{2} F_{2}=\bar{F} \\
& \theta_{4}=\left(w_{1} \lambda_{1}+w_{2} \lambda_{2}\right)\left(\frac{F_{1}}{\lambda_{1}}\right)\left(\frac{F_{2}}{\lambda_{2}}\right)=\bar{\lambda}\left(\frac{F_{1}}{\lambda_{1}}\right)\left(\frac{F_{2}}{\lambda_{2}}\right)
\end{aligned}
$$

and $\bar{F}=w_{1} F_{1}+w_{2} F_{2}$ and $\bar{\lambda}=w_{1} \lambda_{1}+w_{2} \lambda_{2}$ represent the weighted-average blood flow and blood:brain partition coefficient, respectively, in the mixed tissue. Because the four coefficients depend on five independent parameters, namely $F_{1}, \lambda_{1}, F_{2}, \lambda_{2}$, and $w_{1}$, the parameters will not be identifiable unless some additional information is utilized. In the next section we introduce a set of physiological constraints to render the parameters identifiable.

\section{A. Physiological Constraints: The Blood:Brain Partition Coefficient}

When ${ }^{15} \mathrm{O}$-labeled water is used as a blood flow tracer, the tissue:blood partition coefficient for the tracer can be determined from the water contents in brain tissue and whole blood; partition coefficients of 0.98 and $0.82 \mathrm{ml}$ blood $/ \mathrm{g}$ brain have thus been assigned to gray matter and white matter, respectively [21]. For other blood flow tracers, the blood:brain partition coefficient can be measured in equilibrium experiments. Therefore, to reduce the number of parameters to be estimated, we assume that $\lambda_{1}$ and $\lambda_{2}$ are known.

\section{B. The Linear Least Squares Algorithm: Equal Partition Coefficients in the Tissues}

We consider first a hypothetical blood flow tracer which has equal blood:brain partition coefficients in all homogeneous constituents of the mixed tissue, i.e., $\lambda_{1}=\lambda_{2}=\bar{\lambda}$, where $\bar{\lambda}$ is assumed to be known. From (7) we have $\theta_{4}=\bar{\lambda} \theta_{2}$, and this constraint can be incorporated directly into (6) as

$$
\begin{aligned}
C_{i}(T)= & -\theta_{1} \int_{0}^{T} C_{i}(t) d t+\theta_{2}\left(-\int_{0}^{T} \int_{0}^{t} C_{i}(s) d s d t\right. \\
& \left.+\bar{\lambda} \int_{0}^{T} \int_{0}^{t} C_{a}(s) d s d t\right)+\theta_{3} \int_{0}^{T} C_{a}(t) d t .
\end{aligned}
$$

This suggests the following algorithm. Given a series of measurements of the tissue concentration made over the intervals $\left[s_{1}, t_{1}\right],\left[s_{2}, t_{2}\right], \ldots,\left[s_{n}, t_{n}\right]$, form the vector

$$
\mathbf{y}=\left[\begin{array}{llll}
\int_{s_{1}}^{t_{1}} C_{i}(t) d t & \int_{s_{2}}^{t_{2}} C_{i}(t) d t & \cdots & \int_{s_{n}}^{t_{n}} C_{i}(t) d t
\end{array}\right]^{\operatorname{Tr}}
$$

(where $\operatorname{Tr}$ denotes the vector transpose) and the matrix (see (10), shown at the bottom of the next page). Then

$$
\hat{\theta}=\left[\begin{array}{lll}
\hat{\theta_{1}} & \hat{\theta_{2}} & \hat{\theta_{3}}
\end{array}\right]^{\operatorname{Tr}}=\left(\mathbf{X}^{\operatorname{Tr}} \mathbf{H} \mathbf{X}\right)^{-1} \mathbf{X}^{\operatorname{Tr}} \mathbf{H} \mathbf{y}
$$

is the weighted least squares estimate of $\theta$ for the weighting matrix $\mathbf{H}$. $\mathbf{H}$ is usually the inverse of the covariance matrix of 
the errors in the measurements $\mathbf{y}_{\boldsymbol{i}}$. Estimates of the parameters $F_{1}, F_{2}, \bar{F}$, and $w_{1}$ are found by solving (7), i.e.,

$$
\begin{array}{ll}
\hat{F}_{1}=\frac{\lambda_{1}}{2}\left(\hat{\theta}_{1}+\sqrt{\hat{\theta}_{1}^{2}-4 \hat{\theta}_{2}}\right) & \hat{\bar{F}}=\hat{\theta}_{3} \\
\hat{F}_{2}=\frac{\lambda_{2}}{2}\left(\hat{\theta}_{1}-\sqrt{\hat{\theta}_{1}^{2}-4 \hat{\theta}_{2}}\right) & \hat{w}_{1}=\frac{\hat{\theta}_{3}-\hat{F}_{2}}{\hat{F}_{1}-\hat{F}_{2}} .
\end{array}
$$

\section{Additional Physiological Constraints}

Physiological constraints require that the parameters $F_{1}, \lambda_{1}, F_{2}, \lambda_{2}$ be positive and relative tissue weights be nonnegative. Since $w_{2}=1-w_{1}$, this further implies that $0 \leq w_{1} \leq 1$. Rather than implement directly these constraints, which are nonlinear in the coefficients $\theta_{i}$ and would, therefore, introduce a considerable computational burden, we took a somewhat pragmatic approach that assumed that fits that violated these conditions were due to either insufficient heterogeneity in the region (too small a content of either gray or white matter) or noise levels too high to detect physiologically valid parameters from the heterogeneous tissue model. In these cases, the tissue was considered to be indistinguishable from a homogeneous tissue and only weighted-average flow was estimated from $\hat{\theta}_{3}$. Additionally, in order to avoid ill conditioning in the estimate $\hat{w}_{1}$, tissues for which the difference between the estimates $\hat{F}_{1}$ and $\hat{F}_{2}$ was less than $10 \%$ of their mean were arbitrarily classified as homogeneous.

\section{The Algorithm for Unequal Partition Coefficients in the Tissues}

In the case of unequal blood:brain partition coefficients for the two tissues comprising the mixture, i.e., $\lambda_{1} \neq \lambda_{2}, \bar{\lambda}$ is unknown a priori owing to the fact that it depends on the value of $w_{1}$, which is to be estimated. Hence the least squares estimation procedure outlined in the previous section must be modified. Rather than introduce nonlinear constraints, we chose to implement an iterative procedure that starts with an initial estimate of $\bar{\lambda}$ (the mean of $\lambda_{1}$ and $\lambda_{2}$ ), performs the least squares estimation of the parameters to produce new estimates of $w_{1}$ and $\bar{\lambda}$, and repeats until either the percentage change in successive estimates of $\bar{\lambda}$ is below a prespecified tolerance, or a maximum number of iterations is reached. Physiological constraints are imposed only after convergence of $\bar{\lambda}$. As implemented, the tolerance for change in estimates of $\bar{\lambda}$ was set at $0.5 \%$, and a maximum of ten iterations were used.
E. Reducing the Number of Parameters: An Algorithm for Use When White Matter Flow Is Known

Cerebral blood flow in white matter has been shown to be fairly homogeneous in conscious animals [18], [19]; this is most probably true in the human brain as well. Furthermore some white matter regions in the human brain, such as the corpus callosum, are sufficiently large in comparison with the spatial resolution of the PET scanner that it should be possible to measure their blood flow on the basis of a homogeneous tissue model. This suggests the possibility of introducing a further constraint on the system of equations in order to reduce the variance in the estimates of gray matter flow.

The constraint setting the value of white matter flow to a fixed value implies that $\theta_{1}$ and $\theta_{2}$ are not longer independent, but related by

$$
\theta_{2}=\left(\frac{F_{2}}{\lambda_{2}}\right)\left(\theta_{1}-\frac{F_{2}}{\lambda_{2}}\right) .
$$

Substituting (13) into (8) yields

$$
\begin{aligned}
C_{i}(T)+ & \left(\frac{F_{2}}{\lambda_{2}}\right)^{2}\left(-\int_{0}^{T} \int_{0}^{t} C_{i}(s) d s d t\right. \\
+ & \left.\bar{\lambda} \int_{0}^{T} \int_{0}^{t} C_{a}(s) d s d t\right) \\
= & -\theta_{1}\left[\int_{0}^{T} C_{i}(t) d t+\left(\frac{F_{2}}{\lambda_{2}}\right)\right. \\
& \left.\times\left(-\int_{0}^{T} \int_{0}^{t} C_{i}(s) d s d t+\bar{\lambda} \int_{0}^{T} \int_{0}^{t} C_{a}(s) d s d t\right)\right] \\
& +\theta_{3} \int_{0}^{T} C_{a}(t) d t
\end{aligned}
$$

The parmeters $\theta_{1}$ and $\theta_{3}$ are then estimated by defining the vector (see (15) shown at the bottom of the page) the matrix (see (16), shown at the bottom of the page) and solving the linear least squares problem as in the previous sections, i.e.,

$$
\hat{\theta}=\left[\begin{array}{ll}
\hat{\theta}_{1} & \hat{\theta}_{3}
\end{array}\right]^{\operatorname{Tr}}=\left(\mathbf{X}^{\operatorname{Tr}} \mathbf{H X}\right)^{-1} \mathbf{X}^{\operatorname{Tr}} \mathbf{H} \mathbf{y} .
$$

\section{F. Correction for Delay and Dispersion in the Measured Input Function}

Inaccuracies in the measured input function, including delay between the tracer appearance time in the brain and that at the arterial sampling site as well as dispersion of the measured function are well known to lead to systematic errors in rCBF determi-

$$
\mathbf{X}=\left[\begin{array}{ccc}
-\int_{s_{1}}^{t_{1}} \int_{0}^{T} C_{i}(t) d t d T & \int_{s_{1}}^{t_{1}} \int_{0}^{T} \int_{0}^{t}\left\{-C_{i}(s)+\bar{\lambda} C_{a}(s)\right\} d s d t d T & \int_{s_{1}}^{t_{1}} \int_{0}^{T} C_{a}(t) d t d T \\
\vdots & \vdots & \vdots \\
-\int_{s_{n}}^{t_{n}} \int_{0}^{T} C_{i}(t) d t d T & \int_{s_{n}}^{t_{n}} \int_{0}^{T} \int_{0}^{t}\left\{-C_{i}(s)+\bar{\lambda} C_{a}(s)\right\} d s d t d T & \int_{s_{n}}^{t_{n}} \int_{0}^{T} C_{a}(t) d t d T
\end{array}\right]
$$


nations (see, e.g., [22] and [23]). Of the strategies developed to take these factors into account, the multilinear minimization approach for simultaneous estimation of input function delay and dispersion [24] is particularly suited to adaptation for use with the current estimation procedures. Briefly, with this approach the dispersion of the measured input function, $d(t)$, is approximated by a monoexponential function, i.e.,

$$
d(t)=\frac{1}{\tau} e^{(-t / \tau)}
$$

where $\tau$ is the time constant of the dispersion, and a delay of length $\Delta t$ is taken into account by shifting the time scale of of the measured input function, $C_{m}(t)$, so that

$$
C_{a}(t)=C_{m}(t+\Delta t)+\tau \frac{d}{d t} C_{m}(t+\Delta t)
$$

Substituting (19) into (6) yields

$$
\begin{aligned}
C_{i}(T) & \\
= & -\theta_{1} \int_{0}^{T} C_{i}(t) d t-\theta_{2} \int_{0}^{T} \int_{0}^{t} C_{i}(s) d s d t \\
& +\tau \theta_{3} C_{m}(T+\Delta t)+\left(\theta_{3}+\tau \theta_{4}\right) \int_{0}^{T} C_{m}(t+\Delta t) d t \\
& +\theta_{4} \int_{0}^{T} \int_{0}^{t} C_{m}(s+\Delta t) d s d t
\end{aligned}
$$

where $\theta_{1}$ through $\theta_{4}$ are defined in (7). Multilinear minimization consists of considering a range of possible values for $\Delta t$; for each fixed $\Delta t$ the linear least squares problem is solved and resultant weighted residual sum of squares determined. The value of $\Delta t$ for which the residual sum of squares is minimum is chosen, and the best parameter estimates are those corresponding to the chosen $\Delta t$.

When white matter blood flow is known inclusion of the delay and dispersion of the input function in the estimation of gray matter and weighted-average blood flow is accomplished by computing [see (21), shown at the bottom of the previous page] and solving the linear least squares problem as in the previous sections, i.e.,

$$
\hat{\theta}=\left[\begin{array}{lll}
\hat{\theta}_{1} & \hat{\gamma}_{3} & \hat{\gamma}_{4}
\end{array}\right]^{\operatorname{Tr}}=\left(\mathbf{X}^{\operatorname{Tr}} \mathbf{H X}\right)^{-1} \mathbf{X}^{\operatorname{Tr}} \mathbf{H y}
$$

where $\mathrm{y}$ is given in (15). In this case

$$
\hat{F}_{1}=\lambda_{1} \hat{\theta}_{1}-\left(\frac{\lambda_{1}}{\lambda_{2}}\right) F_{2}
$$

$\hat{\theta}_{2}$ is given by (13), $\hat{\tau}$ is the smaller (real) root of $\bar{\lambda} \hat{\theta}_{2} r^{2}-\hat{\gamma}_{4} r+$ $\hat{\gamma}_{3}=0$, and $\hat{\bar{F}}$ is the larger root of $r^{2}-\hat{\gamma}_{4} r+\bar{\lambda} \hat{\theta}_{2} \hat{\gamma}_{3}=0$.

\section{Simulation STUdiES}

Computer simulations were performed with a range of gray matter blood flows, relative tissue compositions, and noise levels in the tissue measurements in order to determine the bias and variance of the estimates of gray matter and weighted-average blood flow. An input function from the literature was used [7]; it represents the activity, corrected for radioactive

$$
\begin{aligned}
& \mathbf{y}=\left[\begin{array}{c}
\int_{s_{1}}^{t_{1}} C_{i}(t) d t+\left(\frac{F_{2}}{\lambda_{2}}\right)^{2} \int_{s_{1}}^{t_{1}} \int_{0}^{T} \int_{0}^{t}\left\{-C_{i}(s)+\bar{\lambda} C_{a}(s)\right\} d s d t d T \\
\vdots \\
\int_{s_{n}}^{t_{n}} C_{i}(t) d t+\left(\frac{F_{2}}{\lambda_{2}}\right)^{2} \int_{s_{n}}^{t_{n}} \int_{0}^{T} \int_{0}^{t}\left\{-C_{i}(s)+\bar{\lambda} C_{a}(s)\right\} d s d t d T
\end{array}\right] \\
& \mathbf{X}=\left[\begin{array}{lll}
-\int_{s_{1}}^{t_{1}} \int_{0}^{T} C_{i}(t) d t d T+\left(\frac{F_{2}}{\lambda_{2}}\right) \int_{s_{1}}^{t_{1}} \int_{0}^{T} \int_{0}^{t}\left\{-C_{i}(s)+\bar{\lambda} C_{a}(s)\right\} d s d t d T & \int_{s_{1}}^{t_{1}} \int_{0}^{T} C_{a}(t) d t d T \\
-\int_{s_{n}}^{t_{n}} \int_{0}^{T} C_{i}(t) d t d T+\left(\frac{F_{2}}{\lambda_{2}}\right) \int_{s_{n}}^{t_{n 2}} \int_{0}^{T} \int_{0}^{t}\left\{-C_{i}(s)+\bar{\lambda} C_{a}(s)\right\} d s d t d T & \vdots \\
\int_{s_{n}}^{t_{n}} \int_{0}^{T} C_{a}(t) d t d T
\end{array}\right]
\end{aligned}
$$

$\left.\begin{array}{l}\mathbf{X}=\int_{s_{1}}^{t_{1}} \int_{0}^{T} C_{i}(t) d t d T+\left(\frac{F_{2}}{\lambda_{2}}\right) \int_{s_{1}}^{t_{1}} \int_{0}^{T} \int_{0}^{t}\left\{-C_{i}(s)+\bar{\lambda} C_{m}(s)\right\} d s d t d T \int_{s_{1}}^{t_{1}} C_{m}(t+\Delta t) d t \int_{s_{1}}^{t_{1}} \int_{0}^{T} C_{m}(t+\Delta t) d t d T \\ \vdots \\ -\int_{s_{n}}^{t_{n}} \int_{0}^{T} C_{i}(t) d t d T+\left(\frac{F_{2}}{\lambda_{2}}\right) \int_{s_{n}}^{t_{n}} \int_{0}^{T} \int_{0}^{t}\left\{-C_{i}(s)+\bar{\lambda} C_{m}(s)\right\} d s d t d T \int_{s_{n}}^{t_{n}} C_{m}(t+\Delta t) d t \int_{s_{n}}^{t_{n}} \int_{0}^{T} C_{m}(t+\Delta t) d t d T\end{array}\right]$ 
A. Arterial Blood Activity

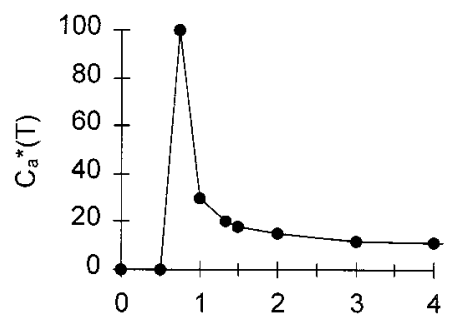

B. Tissue Activity

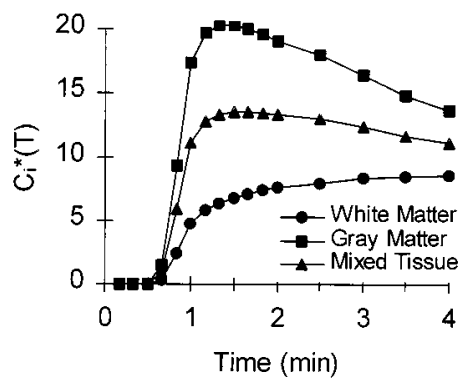

Fig. 1. (A) Input function used in simulations, from [7]. (B) Simulated noise-free concentration of $\mathrm{H}_{2}^{15} \mathrm{O}$ in a heterogeneous tissue that is comprised of $50 \%$ gray matter with $F_{1}=80 \mathrm{ml} / 100 \mathrm{~g} / \mathrm{min}$ and $\lambda_{1}=0.98$, and $50 \%$ white matter with $F_{2}=20 \mathrm{ml} / 100 \mathrm{~g} / \mathrm{min}$ and $\lambda_{2}=0.82$. Input function represents activity, corrected for radioactive decay of the isotope, in whole blood following intravenous bolus injection of $\mathrm{H}_{2}^{15} \mathrm{O}$. Units of activity are arbitrarily scaled.

decay of the isotope, in whole blood following intravenous bolus injection of $\mathrm{H}_{2}^{15} \mathrm{O}$ [Fig. 1(A)]. Tissue activity curves were computed for white matter $\left(F_{2}=20 \mathrm{ml} / 100 \mathrm{~g} / \mathrm{min}\right)$ and gray matter $\left(F_{1}=40,50,60,80,110\right.$, or $\left.120 \mathrm{ml} / 100 \mathrm{~g} \mathrm{~min}\right)$, and their weighted average determined $\left(w_{1}=0.05,0.1,0.2,0.5\right.$, 0.8, 0.9, or 0.95) [Fig. 1(B)]. Blood:brain partition coefficients were those of water, i.e., 0.98 and $0.82 \mathrm{ml}$ blood/g brain for gray and white matter, respectively [21]. The PET scanning schedule was assumed to consist of 12 10-s scans followed by four 30-s scans for a total scanning time of $4 \mathrm{~min}$. Total tissue activity was integrated over each scan interval. Pseudorandom noise was generated from a zero-mean Gaussian distribution to simulate the Poisson noise of counting statistics. Although Poisson statistics strictly apply only to sinogram data, and this noise model (with variance scaled appropriately for the number of resolution elements) applies in reconstructed data only to a uniform disc of activity [25], it was used in the simulations to give an initial indication of the performance of the algorithms. Assuming perfect decay correction, the Thus the noise model used was Gaussian with variance in the nondecay-corrected tissue activity, $\sigma_{k-\text { ndc }}^{2}$, assumed to be proportional to the number of counts collected in each scan interval, i.e.,

$$
\sigma_{k-\mathrm{ndc}}^{2} \equiv \operatorname{var}\left[\int_{t_{k-1}}^{t_{k}} C_{i}(t) e^{-\beta_{t}} d t\right]=\alpha \int_{t_{k-1}}^{t_{k}} C_{i}(t) e^{-\beta_{t}} d t
$$

where $\beta$ is the isotope decay constant for ${ }^{15} \mathrm{O}(0.693 / 122.24$ $\mathrm{s}^{-1}$ ) and $\alpha$ is a proportionality constant.coefficient of variation for the decay-corrected tissue activity is the same as that of the
Coefficient of Variation in Simulated Tissue Activity

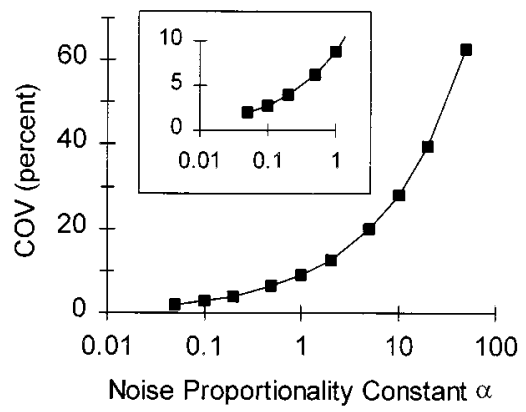

Fig. 2. Average coefficient of variation (COV) of simulated tissue concentration in final four 30-s frames of data, i.e., 2-4 min after bolus injection of isotope, for different noise proportionality constants $\alpha$. Data are for a mixed tissue comprised of $50 \%$ gray matter with $F_{1}=80 \mathrm{ml} / 100 \mathrm{~g} / \mathrm{min}$ and $\lambda_{1}=0.98$, and $50 \%$ white matter with $F_{2}=20 \mathrm{ml} / 100 \mathrm{~g} / \mathrm{min}$ and $\lambda_{2}=$ 0.82 ; COV was similar in other mixed tissues.

nondecay-corrected tissue activity. Therefore, the variance of the decay corrected activity, $\sigma_{k-\mathrm{dc}}^{2}$, is given by

$$
\begin{aligned}
\sigma_{k-\mathrm{dc}}^{2} & \equiv \operatorname{var}\left[\int_{t_{k-1}}^{t_{k}} C_{i}(t) d t\right] \\
& =\alpha \int_{t_{k-1}}^{t_{k}} C_{i}(t) d t\left[\frac{\int_{t_{k-1}}^{t_{k}} C_{i}(t) d t}{\int_{t_{k-1}}^{t_{k}} C_{i}(t) e^{-\beta_{t}} d t}\right] .
\end{aligned}
$$

As can be seen from (25), the variance depends on the absolute units of $C_{i}(t)$, which derive from the units of $C_{a}(t)$ used in the simulation. The relationship between the proportionality constant $\alpha$ and the coefficient of variation in the tissue measurements used in the present study is illustrated in Fig. 2. All programs were written in Matlab Version 5.3.1 (The MathWorks, Inc., Natick, MA) and run on a personal computer (Dell 410 workstation with dual 400-MHz Pentium II processors, Dell Computer, Round Rock, TX). The systems of differential equations were solved with fourth and fifth order Runge-Kutta formulas [26]. Integrals of the simulated measured tissue concentrations were computed by interpolating between measurements by the method of weighted overlapping parabolas [27]. Linear least squares estimations were conducted on data integrated from zero time $\left(s_{k}=0,(9)-(10),(15)-(16)\right)$ and on data integrated over each scan interval $\left(s_{k}=t_{k-1}\right)$. The weighting matrix $\mathbf{H}$ for the linear estimations of (9)-(10) was the diagonal matrix of inverses of the simulated tissue measurements; linear estimations of (15) and either (16) or (21) were unweighted. In the estimations that employed (15)-(16), which assume a fixed value for blood flow in the white matter, $F_{2}$ was set at $90 \%, 100 \%$, or $110 \%$ of its true value. To investigate the effect of total scanning time, additional simulations were performed in which scans terminated at 90, 120, 150, 180,210 , or $240 \mathrm{~s}$. The effect of heterogeneity of gray matter blood flows within a region of interest was investigated by considering simulated tissue regions that included a mixture of two, three, or five gray matter compartments plus a white matter compartment. The distributions of gray matter flows had a mean of $80 \mathrm{ml} / 100 \mathrm{~g} / \mathrm{min}$ and standard deviation of $\sim 20$ 
$\mathrm{ml} / 100 \mathrm{~g} / \mathrm{min}$; the coefficient of variation of $25 \%$ was chosen to produce a distribution similar to that of gray matter $\mathrm{rCBF}$ measured in the cat [18]. In this set of simulations gray matter flows estimated by use of (15)-(16) were compared to the mean gray matter blood flow for the distribution. Simulations were also performed with delays in the input function ranging from -5 to $+5 \mathrm{~s}$ and dispersion time constants of 0,5 , or 10 s. A total of 1000 simulations was performed for each set of parameters, and the mean, variance, and mean square error for each parameter estimate was computed. For comparison, gray matter and weighted-average $\mathrm{rCBF}$ were also computed by a modification of the least squares search algorithm of Koeppe et al. [10] to allow for a weighted mixture of two tissues (Appendix II). Additionally, weighted-average rCBF was computed by three algorithms that are based on a homogeneous tissue model: the least squares search algorithm [10] and the linear least squares algorithm of Feng et al. [14], the latter with and without a constraint fixing the tissue:blood partition coefficient at the arithmetic mean of the gray and white matter values, i.e., $\bar{\lambda}=0.9 \mathrm{ml}$ blood $/ \mathrm{g}$ brain.

\section{RESULTS}

Errors in estimating weighted-average blood flow $\bar{F}$ in a heterogeneous region by use of the homogeneous and heterogeneous tissue models are shown in Figs. 3-5. The greatest biases in $\bar{F}$ occured when a homogeneous tissue model with a fixed partition coefficient was used in the estimation. With this model, one of the greatest underestimations of $\bar{F}$ was found when the two tissues in the mixture were equally weighted [Fig. 3(A)]; note that in this particular case the error cannot be attributed to use of an incorrect value for the weighted-average partition coefficient, as the true weighted-average value for this tissue was used. When both flow and partition coefficient were estimated with the homogeneous tissue model, the partition coefficient was consistently underestimated. For example, at low noise levels in a tissue comprised of 50\% gray matter in which blood flow was four times that of white matter the mean estimated $\bar{\lambda}$ was $0.79 \mathrm{ml} / \mathrm{g}$, a bias of $-13 \%$. The bias in $\bar{F}$ was reduced when both flow and partition coefficient were estimated [Figs. 3(A), 4(A), and 5(A)]. The degree of underestimation of $\bar{F}$ [Fig. 4(A)] and $\bar{\lambda}$ (data not shown) increased as the ratio of gray matter blood flow, $F_{1}$, increased relative to white matter blood flow, $F_{2}$. Biases in estimated $\bar{F}$ were negligible when tissue heterogeneity was taken into account in the kinetic model [Figs. 3(A) and 4(A)], but the variance in the estimates was higher than when homogeneous tissue models were employed [Figs. 3(B) and 4(B)]. Overall, the root mean square (rms) error was smallest with the heterogeneous tissue model [Figs. 3(C) and $4(\mathrm{C})]$. Only at the highest noise level examined were errors in the estimates of $\bar{F}$ with the heterogeneous tissue model greater than those obtained by use of the homogeneous tissue model (Fig. 5).

Errors in estimating gray matter blood flow, $F_{1}$, by use of a heterogeneous tissue model are shown in Fig. 6. Total rms error was lowest, and fractional detection of heterogeneity in the region was highest, when the modified least squares search algorithm was used. Computation time with this algorithm, however,
Est Weighted Average rCBF in Heterogeneous Tissue

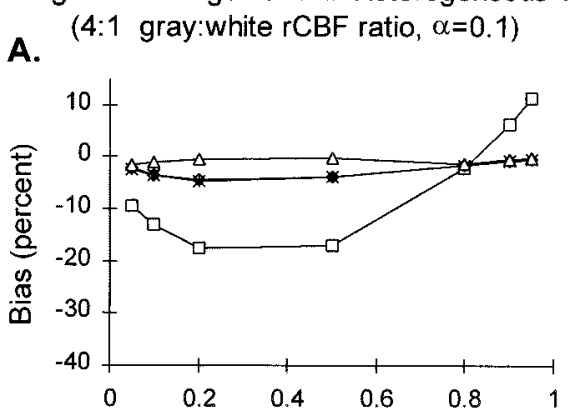

B.

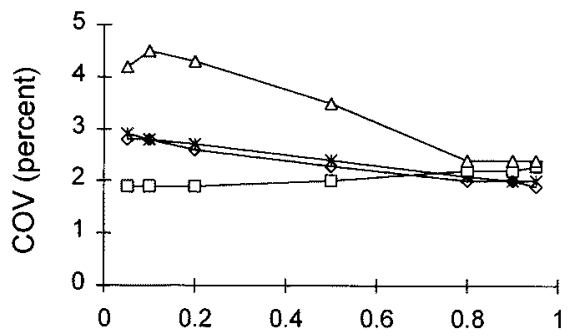

C.
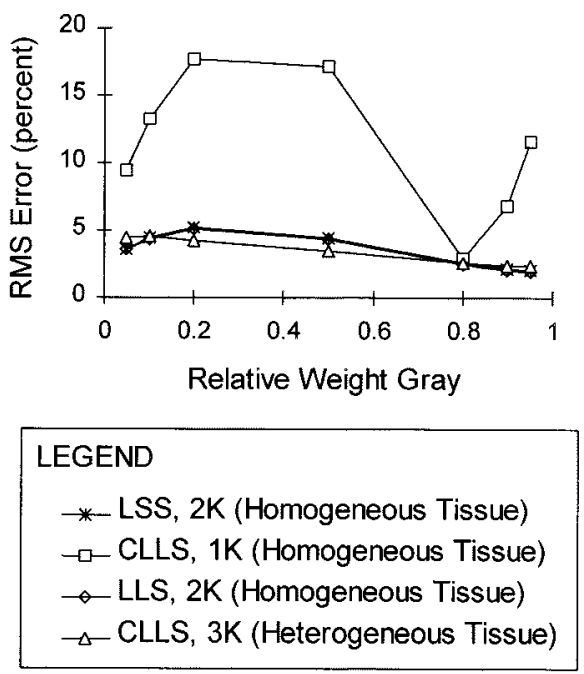

Fig. 3. Errors in estimation of weighted-average blood flow in heterogeneous tissues comprised of various fractions of gray matter. The two-parameter least squares search algorithm (LSS, 2K) [10], which estimates $F$ and $\lambda(x)$, the one-parameter constrained linear least squares algorithm (CLLS, 1K) [14], which estimates only $F$ (squares), and the two-parameter unconstrained linear least squares algorithm (LLS, 2K) [14], which estimates both $F$ and $\lambda$ (diamonds), assume a homogeneous tissue model. The constrained linear least squares algorithm that estimates the three parameters $F_{1}, F_{2}$, and $w_{1}$ (CLLS, 3K) assumes a heterogeneous tissue model (triangles). Blood flows in gray and white matter are $F_{1}=80 \mathrm{ml} / 100 \mathrm{~g} / \mathrm{min}$ and $F_{2}=20 \mathrm{ml} / 100 \mathrm{~g} / \mathrm{min}$, respectively; corresponding partition coefficients are $\lambda_{1}=0.98$ and $\lambda_{2}=$ 0.82 .

was about 500-fold higher than that of the constrained linear least squares algorithms; the former algorithm required an average of 212 min to estimate flow in 1000 simulated regions compared to an average of $0.43 \mathrm{~min}$ for the linear methods. In most cases two to three iterations were required for the linear algorithms. Use of data integrated over each individual frame consistently produced better estimates of $F_{1}$ than use of data integrated from zero time (Fig. 6). Higher ratios of gray matter to white matter blood flow also produced better estimates of $F_{1}$ 
Est Weighted Average rCBF in Heterogeneous Tissue (Gray Matter Weight 50\%, $\alpha=0.1$ )

A.

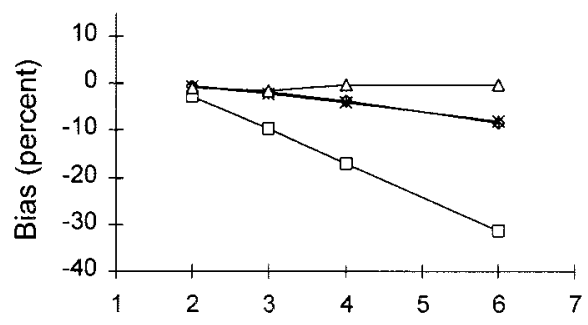

B.

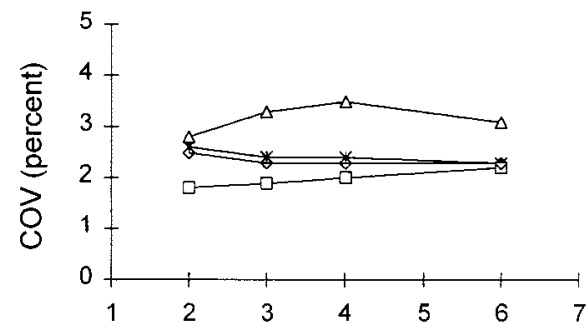

C.

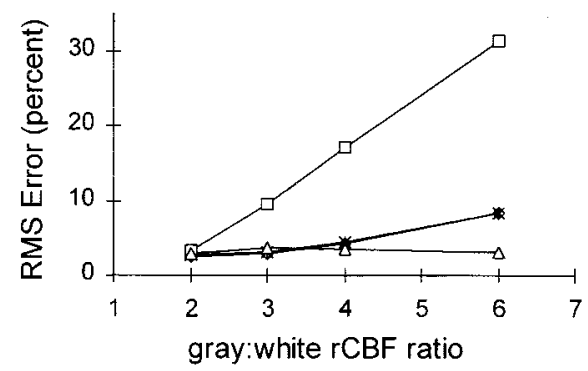

$$
\begin{aligned}
& \text { LEGEND } \\
& \text { * LSS, 2K (Homogeneous Tissue) } \\
& \rightarrow \text { CLLS, 1K (Homogeneous Tissue) } \\
& \rightarrow \text { LLS, 2K (Homogeneous Tissue) } \\
& \triangle \text { CLLS, 3K (Heterogeneous Tissue) }
\end{aligned}
$$

Fig. 4. Errors in estimation of weighted-average blood flow in heterogeneous tissues comprised of 50\% gray matter. The two-parameter least squares search algorithm (LSS, 2K) [10], which estimates $F$ and $\lambda(x)$, the one-parameter constrained linear least squares algorithm (CLLS, 1K) [14], which estimates only $F$ (squares), and the two-parameter unconstrained linear least squares algorithm (LLS, 2K) [14], which estimates both $F$ and $\lambda$ (diamonds), assume a homogeneous tissue model. The constrained linear least squares algorithm that estimates the three parameters $F_{1}, F_{2}$, and $w_{1}$ (CLLS, 3K) assumes a heterogeneous tissue model (triangles). Blood flow in white matter is $F_{2}=$ $20 \mathrm{ml} / 100 \mathrm{~g} / \mathrm{min}$; flows in gray matter are $40,60,80$, or $120 \mathrm{ml} / 100 \mathrm{~g} / \mathrm{min}$. Partition coefficients are $\lambda_{1}=0.98$ and $\lambda_{2}=0.82$ in gray and white matter, respectively.

(data not shown). In tissues that contained $\geq 80 \%$ gray matter, dectection of heterogeneity was poor, and in tissues comprised of $50 \%$ gray matter the fractional rate of detection of heterogeneity decreased by about $50 \%$ as noise levels increased (data not shown). At all noise levels estimates of $F_{1}$ had higher errors than did estimates of $\bar{F}$.

When the linear estimation was further constrained by fixing the value of white matter flow, the estimates of gray matter flow were greatly improved, particularly in tissues that contain at

\section{Est Weighted Average rCBF in Heterogeneous Tissue (Gray Matter Weight 50\%, 4:1 gray:white rCBF ratio)}

A.

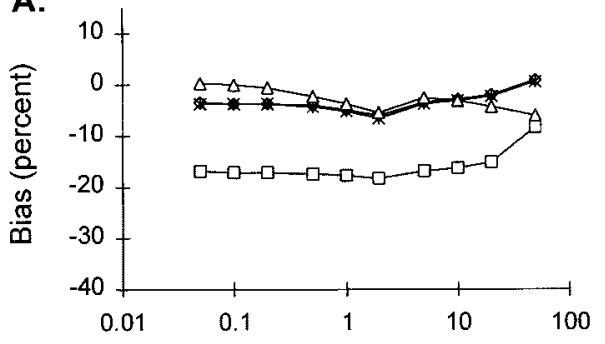

B.

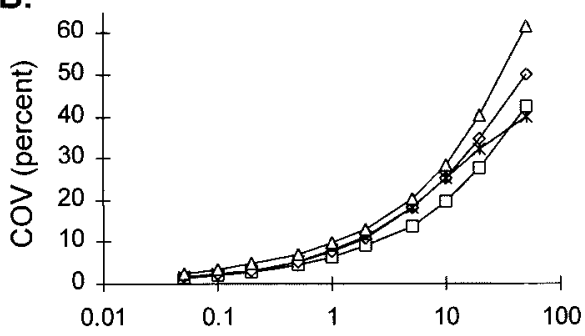

C.

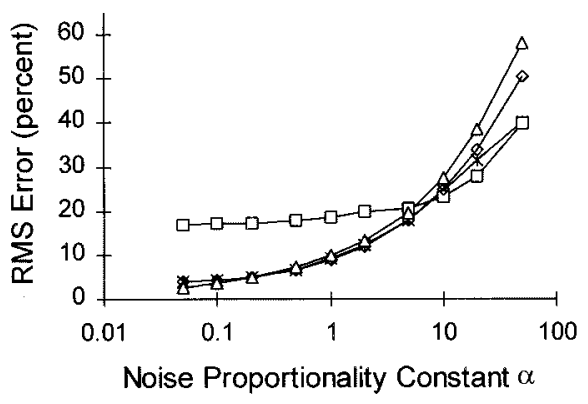

$$
\begin{aligned}
& \text { LEGEND } \\
& \text { * LSS, 2K (Homogeneous Tissue) } \\
& \square \text { - CLLS, 1K (Homogeneous Tissue) } \\
& \rightarrow \text { LLS, 2K (Homogeneous Tissue) } \\
& \triangle \text { CLLS, 3K (Heterogeneous Tissue) }
\end{aligned}
$$

Fig. 5. Errors in estimation of weighted-average blood flow in heterogeneous tissues comprised of $50 \%$ gray matter as a function of noise in the tissue activity. The two-parameter least squares search algorithm (LSS, 2K) [10], which estimates $F$ and $\lambda(x)$, the one-parameter constrained linear least squares algorithm (CLLS, 1K) [14], which estimates only $F$ (squares), and the two-parameter unconstrained linear least squares algorithm (LLS, 2K) [14], which estimates both $F$ and $\lambda$ (diamonds), assume a homogeneous tissue model. The constrained linear least squares algorithm that estimates the three parameters $F_{1}, F_{2}$, and $w_{1}$ (CLLS, 3K) assumes a heterogeneous tissue model (triangles). Blood flows in gray and white matter are $F_{1}=80 \mathrm{ml} / 100 \mathrm{~g} / \mathrm{min}$ and $F_{2}=20 \mathrm{ml} / 100 \mathrm{~g} / \mathrm{min}$, respectively; corresponding partition coefficients are $\lambda_{1}=0.98$ and $\lambda_{2}=0.82$.

least $20 \%$ gray matter (Fig. 7). The errors introduced by performing the analysis with over- or underestimated values of $F_{2}$ is greatest in those tissues that contain the highest proportion of white matter (Fig. 7), but biases were small $(<10 \%)$ in tissues that contain at least 50\% gray matter [see Fig. 7(A)]. Total rms error in the estimate of $F_{1}$ decreased with an increasing flow ratio between gray and white matter (data not shown); at low noise levels it was under $\sim 10 \%$ for a tissue comprised of equal weights gray and white matter whose flow ratios were $\geq 3$. 


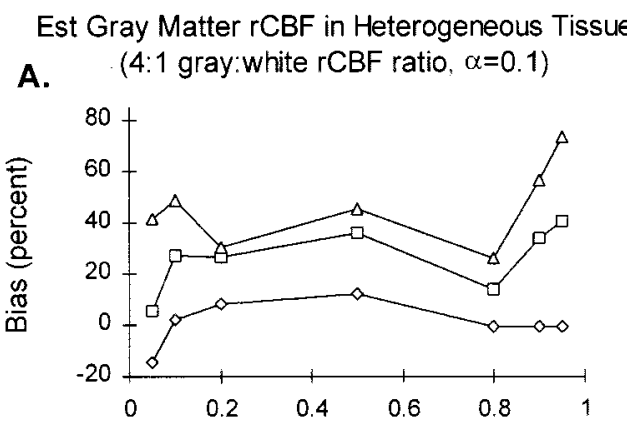

B.

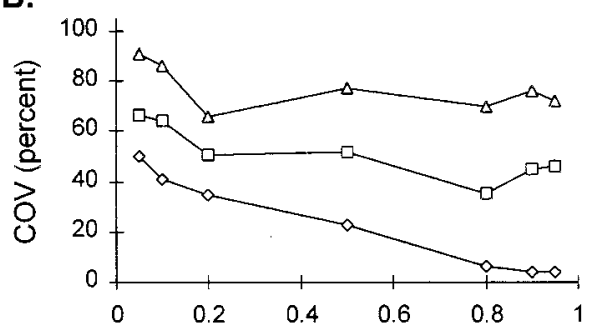

C.

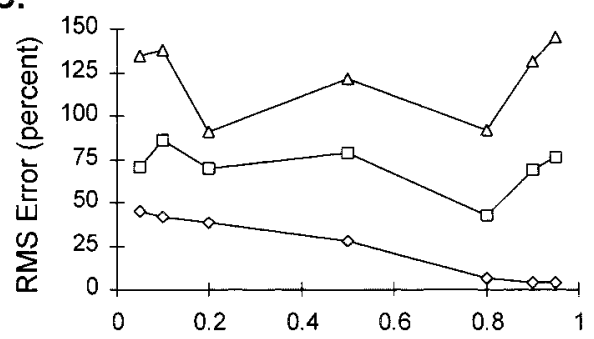

D.

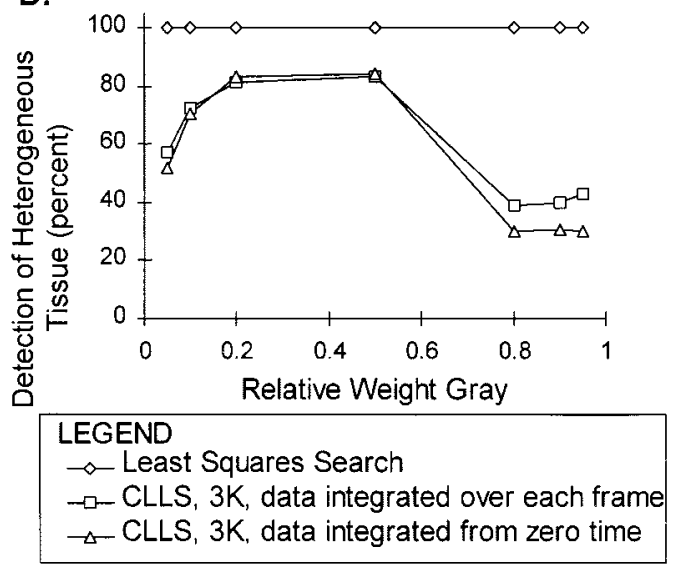

Fig. 6. Errors in estimation of gray matter blood flow in heterogeneous tissues comprised of various fractions of gray matter. The modified least squares search algorithm (Appendix II) (diamonds) is compared to the constrained linear least squares algorithm (CLLS) with data integrated over each scanning frame (squares) or integrated from the start of the experiment (triangles). All algorithms assume a heterogeneous tissue and estimate the three parameters $F_{1}, F_{2}$, and $w_{1}$. Blood flows in gray and white matter are $F_{1}=80 \mathrm{ml} / 100$ $\mathrm{g} / \mathrm{min}$ and $F_{2}=20 \mathrm{ml} / 100 \mathrm{~g} / \mathrm{min}$, respectively; corresponding partition coefficients are $\lambda_{1}=0.98$ and $\lambda_{2}=0.82$.

With increasing noise levels, total rms error in the estimate of $F_{1}$ increased and fractional detection of heterogeneous tissue decreased (Fig. 8). At low noise levels $(\alpha=0.1)$, total rms errors were $4 \%, 7 \%$, or $17 \%$ for tissues comprised of $90 \%, 50 \%$, or $20 \%$ gray matter, respectively [see Fig. 8(C)], and heterogeneity was detected in $100 \%$ of the simulated tissues [see Fig. 8(D)].
Est Gray Matter rCBF in Heterogeneous Tissue (4:1 gray: white $\mathrm{rCBF}$ ratio, $\alpha=0.1$ )

A.

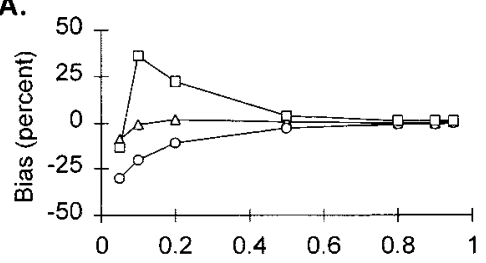

B.

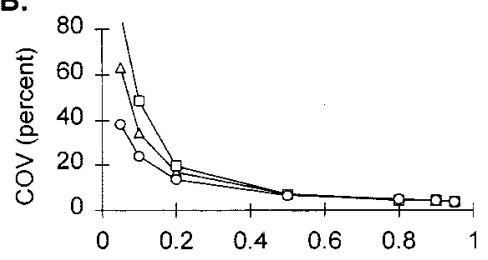

C.

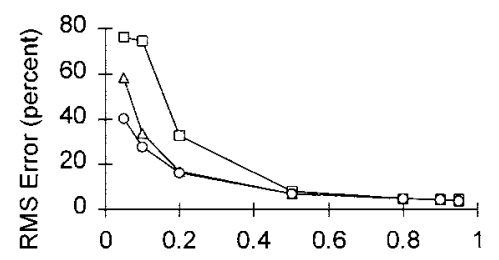

D.

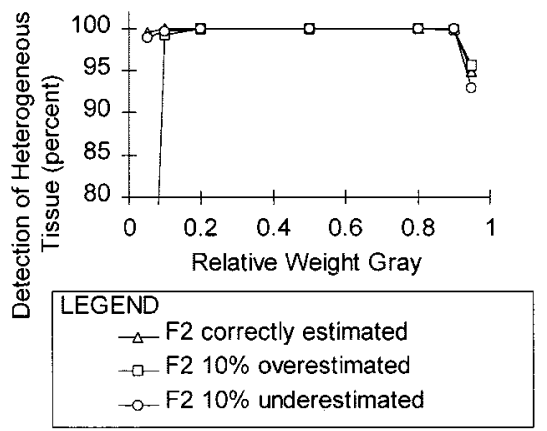

Fig. 7. Errors in estimation of gray matter blood flow with heterogeneous tissue model in which white matter blood flow is known. The constrained linear least squares algorithm with data integrated over each scanning frame was used and assumed values of $F_{2}$ fixed at $100 \%$ (triangles), $110 \%$ (squares), or $90 \%$ (circles) of their true value. Two parameters, $F_{1}$ and $w_{1}$, were estimated. Blood flows in gray and white matter are $F_{1}=80 \mathrm{ml} / 100 \mathrm{~g} / \mathrm{min}$ and $F_{2}=20 \mathrm{ml} / 100$ $\mathrm{g} / \mathrm{min}$, respectively; corresponding partition coefficients are $\lambda_{1}=0.98$ and $\lambda_{2}=0.82$.

At the hightest noise level $(\alpha=50)$, fractional detection of heterogeneous tissue decreased to approximately $51 \%, 66 \%$, or $66 \%$ [see Fig. 8(D)] and total rms error increased to $69 \%, 65 \%$, or $69 \%$ [see Fig. 8(C)] for tissues comprised of $90 \%, 50 \%$, or $20 \%$ gray matter, respectively.

The influence of the total scanning time on the estimates of gray matter flow in a heterogeneous tissue is shown in Fig. 9. Errors in the estimates are little changed by extending the total scanning period from 2 to 4 min after intraveneous administration of the tracer. In predominantly gray matter regions, however, optimal detection of tissue heterogeneity, which is required to obtain an estimate of gray matter flow, requires a total scanning time of not less than 2.5-3 min.

Heterogeneity in gray matter flow had little effect on errors in estimation of $F_{1}$ (Fig. 10) for the symmetric distributions 
Est Gray Matter rCBF in Heterogeneous Tissue (4:1 gray white $\mathrm{CCBF}$ ratio)

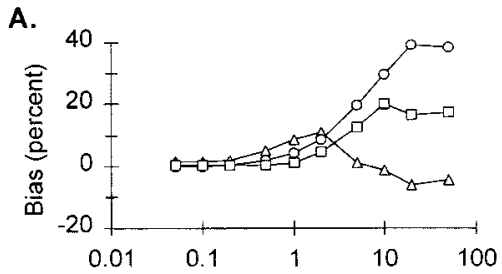

B.

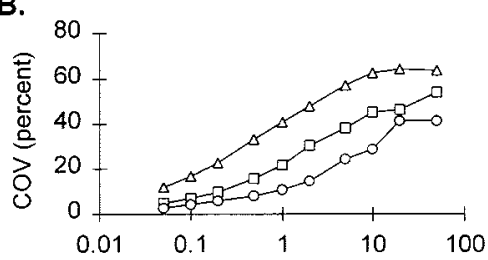

C.

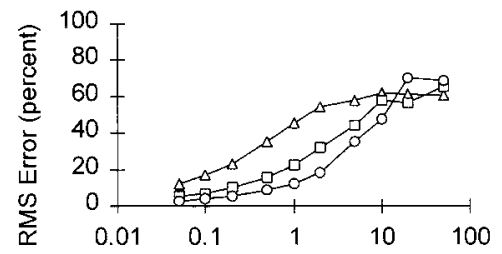

D.

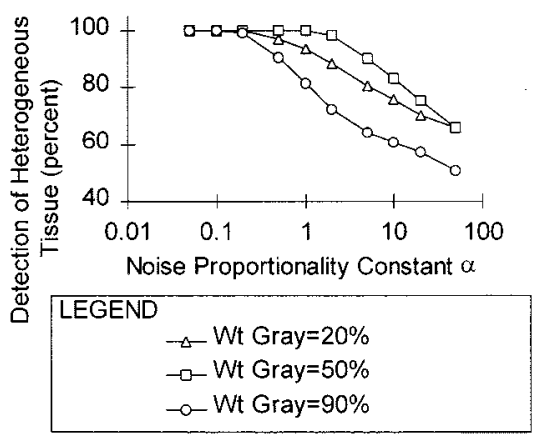

Fig. 8. Errors in estimation of gray matter blood flow with heterogeneous tissue model in which white matter blood flow is known. The effects of increasing noise levels are shown for a mixed tissue that is predominantly white matter (20\% gray, triangles), equal fractions of gray and white matter (50\% gray, squares), or predominantly gray matter (90\% gray, circles). The constrained linear least squares algorithm with data integrated over each scanning frame was used with an assumed value of $F_{2}$ fixed at $100 \%$ of its true value. Two parameters, $F_{1}$ and $w_{1}$, were estimated. Blood flows in gray and white matter are $F_{1}=80 \mathrm{ml} / 100 \mathrm{~g} / \mathrm{min}$ and $F_{2}=20 \mathrm{ml} / 100 \mathrm{~g} / \mathrm{min}$, respectively; corresponding partition coefficients are $\lambda_{1}=0.98$ and $\lambda_{2}=$ 0.82 .

of gray matter flow considered. In tissues comprised of $50 \%$ gray matter, heterogeneity of gray matter flow introduced only a small additional bias compared to a region in which the gray matter flow was uniform [see Fig. 10(A)]. Heterogeneity of gray matter flow had no effect on either the variance in the estimates or the fractional detection of heterogeneity in the tissue as a whole [see Fig. 10(D)].

Fig. 11 illustrates the biases introduced into the estimation of gray matter flow by delay and dispersion of the measured input function relative to the true input function. When no corrections for delay and dispersion were made and only the two parameters $F_{1}$ and $w_{1}$ estimated, large biases in the estimated gray matter flow resulted [see Fig. 11(A)]. When delay and dispersion were
Est Gray Matter rCBF in Heterogeneous Tissue (4:1 gray:white rCBF ratio, $\alpha=0.1$ )

A.

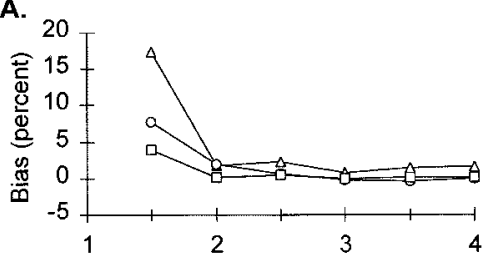

B.

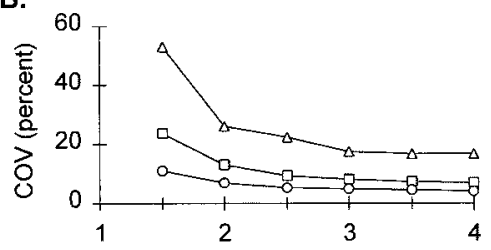

C.

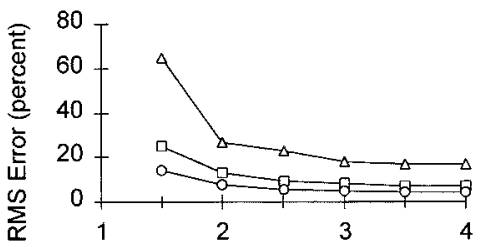

D.

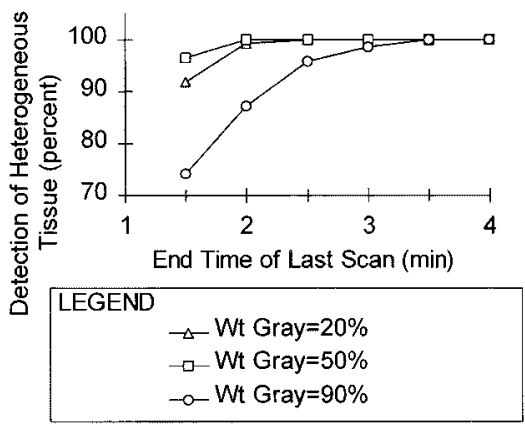

Fig. 9. Errors in estimation of gray matter blood flow with heterogeneous tissue model in which white matter blood flow is known. The effects of terminating the PET scanning process at various times between $90 \mathrm{~s}$ and $4 \mathrm{~min}$ are shown for a mixed tissue that is predominantly gray matter ( $90 \%$ gray, circles), equal fractions gray and white matter (squares), or predominantly white matter (20\% gray, triangles). The constrained linear least squares algorithm with data integrated over each scanning frame was used; $F_{2}$ was fixed at $100 \%$ of its true value. Two parameters, $F_{1}$ and $w_{1}$, were estimated. Blood flows in gray and white matter are $F_{1}=80 \mathrm{ml} / 100 \mathrm{~g} / \mathrm{min}$ and $F_{2}=$ $20 \mathrm{ml} / 100 \mathrm{~g} / \mathrm{min}$, respectively; corresponding partition coefficients are $\lambda_{1}=$ 0.98 and $\lambda_{2}=0.82$.

simultaneously estimated by multilinear minimization, biases in the estimates of gray matter flow were largely eliminated [see Fig. 11(B)]. Estimation of four parameters rather than two, however, led to increases in the coefficients of variation of the estimates from $7 \%-8 \%$ to $9 \%-12 \%$. In general, at all noise levels rms error in the estimates of gray matter flow were somewhat higher and fractional detection of heterogeneity somewhat lower when delay and dispersion of the input function were estimated simultaneously with the estimation of $F_{1}$ and $w_{1}$ (Fig. 12) than when the input function was assumed to have been fully corrected prior to the estimation and only $F_{1}$ and $w_{1}$ were determined (Fig. 8). 


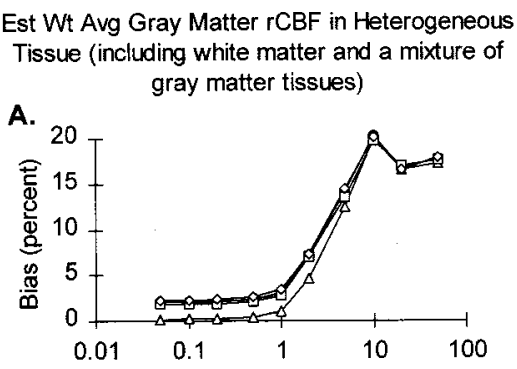

B.

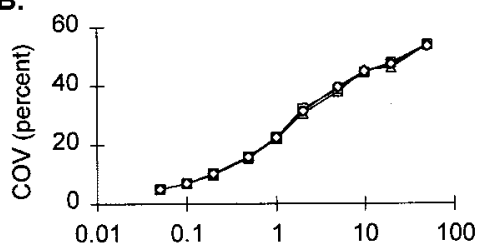

C.

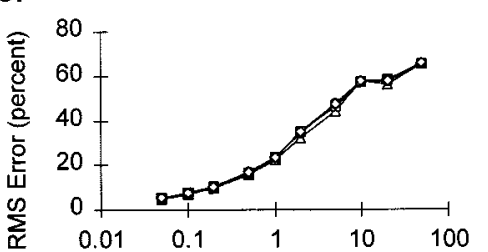

D.

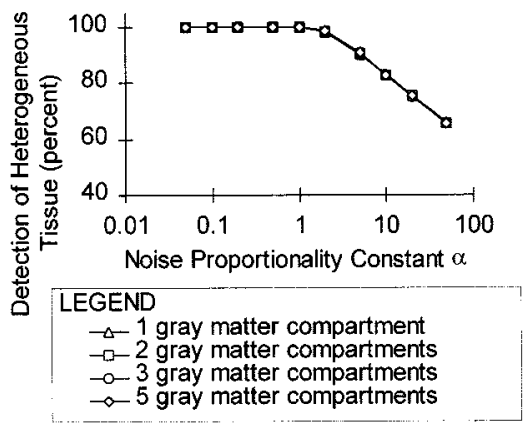

Fig. 10. Errors in estimation of weighted-average gray matter blood flow with heterogeneous tissue model in which white matter blood flow is known The effects of heterogeneity of flow in the gray matter are shown. The total tissue mixture is comprised of $50 \%$ gray matter, $50 \%$ white matter. Gray matter blood flows are either homogeneous (1 gray matter compartment, $F_{1}=80 \mathrm{ml} / 100 \mathrm{~g} / \mathrm{min}$ ), or a heterogeneous mixture of two, three, or five compartments distributed such that the weighted-average gray matter blood flow is $80 \mathrm{ml} / 100 \mathrm{~g} / \mathrm{min}$, and the coefficient of variation of the distribution is $\sim 25 \%$. The two compartment gray matter distribution contains $50 \%$ tissue with a flow of $60 \mathrm{ml} / 100 \mathrm{~g} / \mathrm{min}$, and 50\% tissue with a flow of $100 \mathrm{ml} / 100$ $\mathrm{g} / \mathrm{min}$. The three compartment gray matter distribution is comprised of $22 \%$ tissue with a flow of $50 \mathrm{ml} / 100 \mathrm{~g} / \mathrm{min}, 56 \%$ tissue with a flow of $80 \mathrm{ml} / 100$ $\mathrm{g} / \mathrm{min}$, and $22 \%$ tissue with a flow of $110 \mathrm{ml} / 100 \mathrm{~g} / \mathrm{min}$. The five-compartment distribution is comprised of $8 \%, 20 \%, 44 \%, 20 \%$, and $8 \%$ tissues with flow rates of $40,60,80,100$, and $120 \mathrm{ml} / 100 \mathrm{~g} / \mathrm{min}$, respectively. The constrained linear least squares algorithm with data integrated over each scanning frame was used; $F_{2}$ was fixed at $100 \%$ of its true value. Two parameters, $F_{1}$ and $w_{1}$, were estimated. Blood flow in white matter is $F_{2}=20 \mathrm{ml} / 100 \mathrm{~g} / \mathrm{min}$; partition coefficients in gray and white matter are $\lambda_{1}=0.98$ and $\lambda_{2}=0.82$, respectively.

\section{DISCUSSION}

Many gray matter structures in the human brain are small compared to the spatial resolution of even the most advanced PET scanners currently in use. Particularly in cortical regions, where the gray matter forms a winding band only $2-3 \mathrm{~mm}$ thick, measurements of activity unavoidably include neighboring
Est Gray Matter rCBF in Heterogeneous Tissue (Gray Matter Weight 50\%, 4:1 gray:white rCBF ratio, $\alpha=0.1$ )

A.

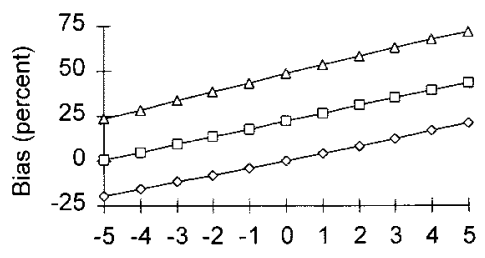

B.

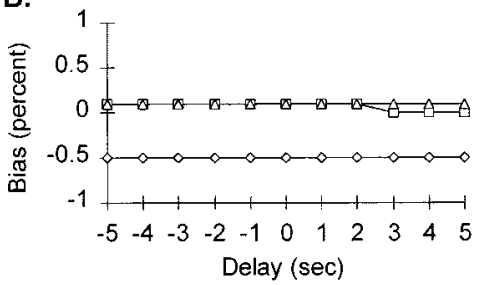

$$
\begin{aligned}
& \text { LEGEND } \\
& \qquad \sim \tau=0 \quad \neg-\tau=5 \quad \rightarrow \tau=10
\end{aligned}
$$

Fig. 11. Bias in estimation of gray matter blood flow in heterogeneous tissue due to delay and dispersion of the measured input function with respect to the true input function. Delay due to timing errors between the measured and true input function range from $-5 \mathrm{~s}$ to $+5 \mathrm{~s}$, and the dispersion time constant of the sampling system, $\tau$, is 0,5 , or $10 \mathrm{~s}$. Tissue is $50 \%$ gray matter. The heterogeneous tissue model, with white matter blood flow assumed to be known, was used in the estimation. In the upper panel (A), no corrections for delay and dispersion were made; two parameters, $F_{1}$ and $w_{1}$, were estimated. Errors in the input function resulted in large biases in the estimated gray matter flow; coefficients of variation in the estimates were $7 \%-8 \%$. In the lower panel (B), the four parameters $F_{1}, w_{1}$, delay, and $\tau$ were determined. Biases in the estimates were removed, but the coefficients of variation in the estimates increased to $9 \%-12 \%$. The constrained linear least squares algorithm with data integrated over each scanning frame was used; $F_{2}$ was fixed at $100 \%$ of its true value. Blood flows in gray and white matter are $F_{1}=80 \mathrm{ml} / 100 \mathrm{~g} / \mathrm{min}$ and $F_{2}=20 \mathrm{ml} / 100 \mathrm{~g} / \mathrm{min}$, respectively; corresponding partition coefficients are $\lambda_{1}=0.98$ and $\lambda_{2}=0.82$.

white matter tissue leading to the well-known partial volume effect. Because blood flow in some cortical regions may be as much as sixfold higher than in neighboring white matter [18], weighted-average blood flow in the mixed tissue underestimates gray matter flow. Furthermore, when the kinetic model used to estimate flow fails to take into account the tissue heterogeneity, weighted-average flow itself is underestimated. Previous simulation studies, which considered the effects of heterogeneity but not noise in the data, found that the underestimation of weighted-average flow was small [2], [4], [7], [10]-[12], [15]-[17]. In the present study, we have expanded these investigations to include the influence of noise in the data, and confirmed that errors in weighted-average flow tend to be small. This is partially due to the characteristics of the tissue:blood partition coefficients used in the current study, i.e., those of water. When a heterogeneous tissue that has two compartments with efflux rate constants $\left(F_{1} / \lambda_{1}\right)$ and $\left(F_{2} / \lambda_{2}\right)$ is approximated by a homogeneous tissue with an efflux rate constant of $(F / \lambda)$, in general the greater the separation in the efflux rate constants in the mixed tissue, the greater the errors due to assuming a single efflux rate constant. For water, flow ratios of $4: 1$ result in an efflux rate constant ratio of 3.3:1 $(=(4 / 0.98) /(1 / 0.82))$. For tracers that have partition coefficients in gray matter less than 
Est Gray Matter rCBF in Heterogeneous Tissue (4:1 gray:white $\mathrm{rCBF}$ ratio, $\Delta \mathrm{t}=5 \mathrm{sec}, \tau=10 \mathrm{sec}$ )

A.

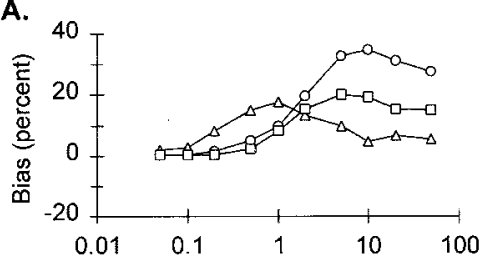

B.

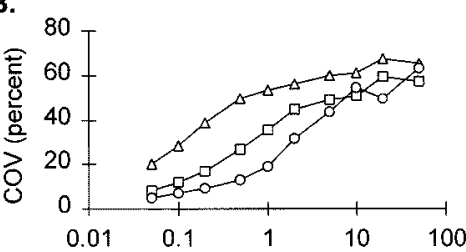

C.

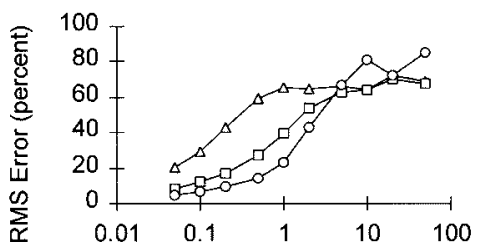

D.

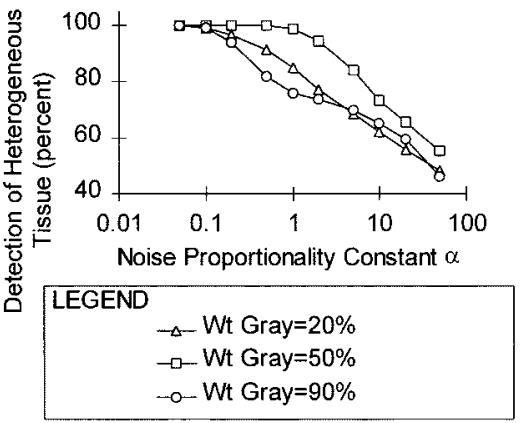

Fig. 12. Errors in estimation of gray matter blood flow due to increased noise levels in the tissue activity data, in the presence of delay and dispersion in the measured input function. Delay due to timing errors between the measured and true input function, $\Delta t$, was assumed to be $5 \mathrm{~s}$, and the dispersion time constant of the sampling system, $\tau, 10 \mathrm{~s}$. The mixed tissue was predominantly white matter (20\% gray, triangles), equal fractions of gray and white matter (50\% gray, squares), or predominantly gray matter ( $90 \%$ gray, circles). The heterogeneous tissue model, with white matter blood flow assumed to be known, was used with simultaneous estimation of $\Delta t$ and $\tau . F_{2}$ was fixed at $100 \%$ of its true value. Blood flows in gray and white matter are $F_{1}=80 \mathrm{ml} / 100 \mathrm{~g} / \mathrm{min}$ and $F_{2}=$ $20 \mathrm{ml} / 100 \mathrm{~g} / \mathrm{min}$, respectively; corresponding partition coefficients are $\lambda_{1}=$ 0.98 and $\lambda_{2}=0.82$. Compare with Fig. 8 in which there were no delays and dispersions of the input function.

or equal to those in white matter, the effects of assuming a homogeneous tissue model in a heterogeneous tissue are greater. A second factor ameliorating the impact of heterogeneity on the estimates of weighted-average flow is the high degree of correlation between the estimates of $F$ and $\lambda$; their simultaneous estimation leads to smaller errors in the estimated weighted-average flow than when $\lambda$ is fixed, even when it is fixed at its true weighted-average value in the mixed tissue. We also observed that the values of $\lambda$ estimated by use of a homogeneous tissue model were smaller than those derived on the basis of the water content of the tissues; our estimates were consistent with the estimates of whole brain partition coefficient in human studies [20]. When the tissue heterogeneity model was used, weighted-average flow estimates were unbiased, but the inclusion of an additional parameter in the model led to somewhat higher variances in the estimates than when a homogeneous tissue model was used.

Use of a kinetic model that assumes a homogeneous tissue, though it does not result in excessive biases in estimates of weighted-average blood flow, does not permit the estimation of flow in the gray matter. For this task a heterogeneous tissue model is required, and the simplest model of a heterogeneous tissue as a weighted mixture of two homogeneous tissues was chosen. This model has been previously used to investigate the errors in weighted-average flow in a mixed tissue estimated with several algorithms that assume a homogeneous tissue model, and reports of $\mathrm{H}_{2}^{15} \mathrm{O}$ PET studies in monkeys [28] and in humans [29] have shown that the model can be used to estimate gray matter flow in large regions of interest with nonlinear least squares fitting. We have found that it is difficult to determine the parameters of the heterogeneous tissue model with nonlinear least squares algorithms, i.e., the final estimates are heavily dependent on initial values of the parameters, and often the algorithms do not converge; this is probably due to the low sensitivity of total tissue activity to changes in white matter flow in regions that contain a significant fraction of gray matter. Additionally, nonlinear least squares algorithms require long computation times. In the present study, we sought to avoid these problems by working with linear least squares algorithms. The linear least squares algorithms used in this study were found to be approximately 500 times faster than the least squares search algorithm [10]. Use of the constrained linear least squares algorithm to estimate three parameters, however, resulted in biased estimates of gray matter flow; the biases were larger in tissues that contained larger fractions of white matter and in tissues with lower ratios of gray matter to white matter blood flow. The biases in the estimates of gray matter flow were almost entirely eliminated when the additional constraint fixing the value of white matter blood flow was included in the linear least squares estimation.

The heterogeneity model used in the current study assumes homogeneity of flow in the gray matter component of the mixed tissue. Yet gray matter flows in brain are themselves quite heterogeneous. In the unanesthetized cat, for example, except for one outlying small structure, gray matter $\mathrm{rCBF}$ ranged from approximately 2-6 times (mean 4.1) that of white matter; the standard deviation of the distribution was $\sim 0.26$ times the mean [18]. When various symmetric gray matter rCBF distributions with these characteristics were simulated, good estimates of weighted-average gray matter flow were obtained despite the model assumption of homogeneity of gray matter flow. Accuracy of the estimates remains to be determined if a specific tissue region is found in which gray matter flow heterogeneity follows another distribution, e.g., skewed, with a larger variance, or with a greater overlap with white matter flow.

The rapid computational speed of the linear algorithms compared with their nonlinear counterparts suggests that these algorithms may be suitableforapplication at the pixellevel. Thehighest noise level examined in the present study corresponded to a coefficient of variation in the final 30-s scans of approximately $65 \%$, which is on the order of the noise levels one might expect in un- 
smoothed pixel level data [30]. At this noise level the coefficients of variation of estimates of both weighted average and gray matter flow reached $40 \%-60 \%$, and the detection of heterogeneity fell off by as much as $50 \%$. The high degree of variability in the estimates calls for caution in applying all blood flow calculation methods to unsmoothed pixel data, and suggests that application to less noisy region of interest data may be more appropriate.

The present study was based on the use of a tracer that is freely diffusible across the blood brain barrier. Although partition coefficients for water were used in the simulations in this study because $\mathrm{H}_{2}^{15} \mathrm{O}$ is the most-widely used PET blood flow tracer and is generally treated as freely diffusible, the results would be expected to apply to other freely diffusible blood flow tracers such as $\left[{ }^{15} \mathrm{O}\right]$ butanol [31], $\left[{ }^{11} \mathrm{C}\right]$ butanol [32], or $\left[{ }^{18} \mathrm{~F}\right]$ fluoromethane [33] with appropriately substituted partition coefficients. Possible diffusion limitations of these tracers were beyond the scope of the current study. The present study has also omitted consideration of intravascular activity included in the PET data and its effect on estimation of rCBF. Additionally, a tracer that is freely diffusible across the blood-brain barrier may also diffuse from brain regions that have a high concentration to regions with a lower concentration during the course of the experimental period. This effect was not considered in the present study and is the subject of further investigation.

\section{APPENDIX I}

\section{DERIVATION OF EQUATION FOR MEASUREMENT OF CEREBRAL} Blood Flow in a Heterogeneous Tissue

Assume the model for distribution of a freely diffusible tracer into a mixed tissue comprised of two homogeneous tissues is given by the set of equations:

$$
\begin{aligned}
& \frac{d C_{i 1}}{d t}=-\left(\frac{F_{1}}{\lambda_{1}}\right) C_{i 1}(t)+F_{1} C_{a}(t) \\
& \frac{d C_{i 2}}{d t}=-\left(\frac{F_{2}}{\lambda_{2}}\right) C_{i 2}(t)+F_{2} C_{a}(t)
\end{aligned}
$$

where

$C_{i 1}$ and $C_{i 2} \quad$ concentrations of the tracer in tissues 1 and 2; $F_{1}$ and $F_{2} \quad$ blood flow per unit mass of tissues 1 and 2;

$\lambda_{1}$ and $\lambda_{2}$ respective tissue:blood partition coefficients for the tracer and tissues in question;

$C_{a} \quad$ concentration of tracer in the arterial blood.

The concentration of tracer in the mixed tissue is the weighted sum of the concentrations in the two homogeneous tissues comprising the mixture, i.e.,

$$
C_{i}(T)=w_{1} C_{i 1}(T)+w_{2} C_{i 2}(T)
$$

$w_{1}$ and $w_{2}=1-w_{1}$ are the relative tissue weights. Differentiating (A.2) we have

$$
\begin{aligned}
\frac{d C_{i}}{d t} & =w_{1} \frac{d C_{i 1}}{d t}+w_{2} \frac{d C_{i 2}}{d t} \\
& =-\left(\frac{w_{1} F_{1}}{\lambda_{1}}\right) C_{i 1}(t)-\left(\frac{w_{2} F_{2}}{\lambda_{2}}\right) C_{i 2}(t)+\bar{F} C_{a}(t)
\end{aligned}
$$

which, after rearrangement, becomes

$$
\left(\frac{w_{1} F_{1}}{\lambda_{1}}\right) C_{i 1}(t)+\left(\frac{w_{2} F_{2}}{\lambda_{2}}\right) C_{i 2}(t)=-\frac{d C_{i}}{d t}+\bar{F} C_{a}(t)
$$

where $\bar{F}=w_{1} F_{1}+w_{2} F_{2}$ is the weighted-average blood flow to the mixed tissue. A second differentiation of (A.2) produces

$$
\begin{aligned}
\frac{d^{2} C_{i}}{d t^{2}}= & w_{1} \frac{d^{2} C_{i 1}}{d t^{2}}+w_{2} \frac{d^{2} C_{i 2}}{d t^{2}}=-\left(\frac{w_{1} F_{1}}{\lambda_{1}}\right) \frac{d C_{i 1}}{d t} \\
& -\left(\frac{w_{2} F_{2}}{\lambda_{2}}\right) \frac{d C_{i 2}}{d t}+\bar{F} \frac{d C_{a}}{d t} \\
= & \left(\frac{w_{1} F_{1}^{2}}{\lambda_{1}^{2}}\right) C_{i 1}(t)+\left(\frac{w_{2} F_{2}^{2}}{\lambda_{2}^{2}}\right) C_{i 2}(t) \\
& -\left(\frac{w_{1} F_{1}^{2}}{\lambda_{1}}+\frac{w_{2} F_{2}^{2}}{\lambda_{2}}\right) C_{a}(t)+\bar{F} \frac{d C_{a}}{d t} .
\end{aligned}
$$

Substituting the identities

$$
\left(\frac{F_{1}^{2}}{\lambda_{1}^{2}}\right)=\left(\frac{F_{1}}{\lambda_{1}}+\frac{F_{2}}{\lambda_{2}}\right)\left(\frac{F_{1}}{\lambda_{1}}\right)-\left(\frac{F_{1} F_{2}}{\lambda_{1} \lambda_{2}}\right)
$$

and

$$
\left(\frac{F_{2}^{2}}{\lambda_{2}^{2}}\right)=\left(\frac{F_{1}}{\lambda_{1}}+\frac{F_{2}}{\lambda_{2}}\right)\left(\frac{F_{2}}{\lambda_{2}}\right)-\left(\frac{F_{1} F_{2}}{\lambda_{1} \lambda_{2}}\right)
$$

into (A.5) we obtain

$$
\begin{aligned}
\frac{d^{2} C_{i}}{d t^{2}} & \\
= & \left(\frac{F_{1}}{\lambda_{1}}+\frac{F_{2}}{\lambda_{2}}\right)\left[\left(\frac{w_{1} F_{1}}{\lambda_{1}}\right) C_{i 1}(t)+\left(\frac{w_{2} F_{2}}{\lambda_{2}}\right) C_{i 2}(t)\right] \\
& -\left(\frac{F_{1} F_{2}}{\lambda_{1} \lambda_{2}}\right)\left[w_{1} C_{i 1}(t)+w_{2} C_{i 2}(t)\right] \\
& -\left(\frac{w_{1} F_{1}^{2}}{\lambda_{1}}+\frac{w_{2} F_{2}^{2}}{\lambda_{2}}\right) C_{a}(t)+\bar{F} \frac{d C_{a}}{d t}
\end{aligned}
$$

Finally, substituting (A.2) and (A.4) into (A.6) and simplifying produces

$$
\begin{aligned}
\frac{d^{2} C_{i}}{d t^{2}}= & -\left(\frac{F_{1}}{\lambda_{1}}+\frac{F_{2}}{\lambda_{2}}\right) \frac{d C_{i}}{d t}-\left(\frac{F_{1} F_{2}}{\lambda_{1} \lambda_{2}}\right) C_{i}(t) \\
& +\bar{F} \frac{d C_{a}}{d t}+\left(\frac{F_{1} F_{2}}{\lambda_{1} \lambda_{2}}\right) \bar{\lambda} C_{a}(t)
\end{aligned}
$$

where $\bar{\lambda}=w_{1} \lambda_{1}+w_{2} \lambda_{2}$ is the weighted-average blood : brain partition coefficient in the mixed tissue. Noting the initial conditions that $C_{a}(t)$ and $C_{i}(t)$, as well as their first derivatives, are zero at $t=0$, twice integrating (A.7) yields the integral equation

$$
\begin{aligned}
C_{i}(T)= & -\theta_{1} \int_{0}^{T} C_{i}(t) d t-\theta_{2} \int_{0}^{T} \int_{0}^{t} C_{i}(s) d s d t \\
& +\theta_{3} \int_{0}^{T} C_{a}(t) d t+\theta_{4} \int_{0}^{T} \int_{0}^{t} C_{a}(s) d s d t
\end{aligned}
$$


where

$$
\begin{aligned}
& \theta_{1}=\left(\frac{F_{1}}{\lambda_{1}}\right)+\left(\frac{F_{2}}{\lambda_{2}}\right) \\
& \theta_{2}=\left(\frac{F_{1}}{\lambda_{1}}\right)\left(\frac{F_{2}}{\lambda_{2}}\right) \\
& \theta_{3}=w_{1} F_{1}+w_{2} F_{2}=\bar{F} \\
& \theta_{4}=\left(w_{1} \lambda_{1}+w_{2} \lambda_{2}\right)\left(\frac{F_{1}}{\lambda_{1}}\right)\left(\frac{F_{2}}{\lambda_{2}}\right)=\bar{\lambda}\left(\frac{F_{1}}{\lambda_{1}}\right)\left(\frac{F_{2}}{\lambda_{2}}\right) .
\end{aligned}
$$

\section{APPENDIX II}

\section{Adaptation of the Least Squares SEARCh Algorithm FOR USE WiTh A HETEROGENEOUS TISSUE MODEL}

The least squares search algorithm [10] is based on a homogeneous tissue model which assumes that the tissue concentration can be described by (2), namely

$$
C_{i}(T)=F \int_{0}^{T} C_{a}(t) e^{-(F / \lambda)(T-t)} d t
$$

where

$C_{i} \quad$ tissue concentration of tracer;

$C_{a}$ arterial input function;

$F \quad$ blood flow per unit weight tissue;

$\lambda$ brain:blood partition coefficient for the tracer and tissue in question.

Given a series of measurements of tissue concentration accumulated over the intervals $\left[t_{0}, t_{1}\right],\left[t_{1}, t_{2}\right], \ldots\left[t_{n-1}, t_{n}\right]$, and an arterial input function, $C_{a}(t)$, the algorithm precomputes the integrals

$$
\begin{aligned}
& \int_{t_{0}}^{t_{1}} \int_{0}^{t} C_{a}(s) e^{-k_{2}^{(j)}(t-s)} d s d t \\
& \int_{t_{1}}^{t_{2}} \int_{0}^{t} C_{a}(s) e^{-k_{2}^{(j)}(t-s)} d s d t \cdots \\
& \int_{t_{n-1}}^{t_{n_{2}}} \int_{0}^{t} C_{a}(s) e^{-k_{2}^{(j)}(t-s)} d s d t
\end{aligned}
$$

for a range of values of $k_{2}^{(j)}$ that covers the expected range of values of $(F / \lambda)$ in the tissue. Then, for each region or pixel, for each value of $k_{2}^{(j)}$, the linear least squares problem $\mathbf{y}=$ $\mathbf{X}^{(j)} \theta^{(j)}$ is solved, where

$$
\begin{aligned}
\mathbf{y}= & {\left[\int_{t_{0}}^{t_{1}} C_{i}(t) d t \int_{t_{1}}^{t_{2}} C_{i}(t) d t \cdots \int_{t_{n-1}}^{t_{n}} C_{i}(t) d t\right]_{(\mathrm{A} .12)}^{\operatorname{Tr}} } \\
\mathbf{X}^{(j)}= & {\left[\int_{t_{0}}^{t_{1}} \int_{0}^{t} C_{a}(s) e^{-k_{2}^{(j)}(t-s)} d s d t\right.} \\
& \int_{t_{1}}^{t_{2}} \int_{0}^{t} C_{a}(s) e^{-k_{2}^{(j)}(t-s)} d s d t \cdots \\
& \left.\int_{t_{n-1}}^{t_{n}} \int_{0}^{t} C_{a}(s) e^{-k_{2}^{(j)}(t-s)} d s d t\right]^{\operatorname{Tr}}
\end{aligned}
$$

and

$$
\theta^{(j)}=\left[K_{1}^{(j)}\right]
$$

The index $j$ is chosen for which $\left\|\mathbf{y}-\mathbf{X}^{(j)} \theta^{(j)}\right\|_{2}^{2}$ is minimized. The estimates of $F$ and $\lambda$ are then given by

$$
\hat{F}=K_{1}^{(j)} \quad \text { and } \quad \hat{\lambda}=\left(\frac{K_{1}^{(j)}}{k_{2}^{(j)}}\right)
$$

To modify this algorithm to allow for a heterogeneous tissue, we assume that the tissue is comprised of a weighted sum of two homogeneous tissues so that

$$
\begin{aligned}
C_{i}(T)= & w_{1} F_{1} \int_{0}^{T} C_{a}(t) e^{-\left(F_{1} / \lambda_{1}\right)(T-t)} d t \\
& +\left(1-w_{1}\right) F_{2} \int_{0}^{T} C_{a}(t) e^{-\left(F_{2} / \lambda_{2}\right)(T-t)} d t
\end{aligned}
$$

$$
\begin{aligned}
\mathbf{y}= & {\left[\begin{array}{c}
\int_{t_{0}}^{t_{1}} C_{i}(t) d t-\lambda_{2} k_{2}^{(k)} \int_{t_{0}}^{t_{1}} \int_{0}^{t} C_{a}(s) e^{-k_{2}^{(k)}(t-s)} d s d t \\
\int_{t_{1}}^{t_{2}} C_{i}(t) d t-\lambda_{2} k_{2}^{(k)} \int_{t_{1}}^{t_{2}} \int_{0}^{t} C_{a}(s) e^{-k_{2}^{(k)}(t-s)} d s d t \\
\vdots \\
\int_{t_{n-1}}^{t_{n}} C_{i}(t) d t-\lambda_{2} k_{2}^{(k)} \int_{t_{n-1}}^{t_{n}} \int_{0}^{t} C_{a}(s) e^{-k_{2}^{(k)}(t-s)} d s d t
\end{array}\right] } \\
\mathbf{X}^{(j k)}= & {\left[\begin{array}{c}
\lambda_{1} k_{2}^{(j)} \int_{t_{0}}^{t_{1}} \int_{0}^{t} C_{a}(s) e^{-k_{2}^{(j)}(t-s)} d s d t-\lambda_{2} k_{2}^{(k)} \int_{t_{0}}^{t_{1}} \int_{0}^{t} C_{a}(s) e^{-k_{2}^{(k)}(t-s)} d s d t \\
\lambda_{1} k_{2}^{(j)} \int_{t_{1}}^{t_{2}} \int_{0}^{t} C_{a}(s) e^{-k_{2}^{(j)}(t-s)} d s d t-\lambda_{2} k_{2}^{(k)} \int_{t_{1}}^{t_{2}} \int_{0}^{t} C_{a}(s) e^{-k_{2}^{(k)}(t-s)} d s d t \\
\lambda_{1} k_{2}^{(j)} \int_{t_{n-1}}^{t_{n}} \int_{0}^{t} C_{a}(s) e^{-k_{2}^{(j)}(t-s)} d s d t-\lambda_{2} k_{2}^{(k)} \int_{t_{n-1}}^{t_{n}} \int_{0}^{t} C_{a}(s) e^{-k_{2}^{(k)}(t-s)} d s d t
\end{array}\right] }
\end{aligned}
$$

and

$$
\theta^{(j k)}=\left[w_{1}^{(j k)}\right]
$$


where

$F_{1}$ and $F_{2} \quad$ blood flows in Tissues 1 and 2;

$\lambda_{1}$ and $\lambda_{2} \quad$ corresponding brain:blood partition coefficients;

$w_{1} \quad$ fractional weight of Tissue 1 .

Let $k_{2 a}$ and $k_{2 b}$ be the efflux rate constants from Tissues 1 and 2, respectively, i.e., $k_{2 a}=\left(F_{1} / \lambda_{1}\right)$ and $k_{2 b}=\left(F_{2} / \lambda_{2}\right)$. Inserting these constants into (A.16) we obtain

$$
\begin{aligned}
C_{i}(T)= & w_{1}\left(\lambda_{1} k_{2 a} \int_{0}^{T} C_{a}(t) e^{-k_{2 a}(T-t)} d t\right. \\
& \left.\quad-\lambda_{2} k_{2 b} \int_{0}^{T} C_{a}(t) e^{-k_{2 b}(T-t)} d t\right) \\
+ & \lambda_{2} k_{2 b} \int_{0}^{T} C_{a}(t) e^{-k_{2 b}(T-t)} d t
\end{aligned}
$$

We assume that $\lambda_{1}$ and $\lambda_{2}$ are known. The algorithm begins by precomputing the integrals, as in (A.11). Then, for each region or pixel, for each pair of values $\left(k_{2}^{(j)}, k_{2}^{(k)}\right)$ the linear least squares problem $y=X^{(j k)} \theta^{(j k)}$ is solved, where [see (A.18)-(A.20) at the bottom of the previous page]. Indexes and $k$ are chosen for which $\left\|\mathbf{y}-\mathbf{X}^{(j k)} \theta^{(j k)}\right\|_{2}^{2}$ is minimized. Then the estimates of $F_{1}, F_{2}$, and $w_{1}$ are given by

$$
\hat{F}_{1}=\lambda_{1} k_{2}^{(j)}, \quad \hat{F}_{2}=\lambda_{2} k_{2}^{(k)}, \quad \text { and } \quad \hat{w}_{1}=\theta^{(j k)} \text {. }
$$

Note that because we assume that $F_{1}>F_{2}$ only those indexes for which $\lambda_{1} k_{2}^{(j)}>\lambda_{2} k_{2}^{(k)}$ need to be examined.

\section{REFERENCES}

[1] S. S. Kety, "The theory and application of the exchange of inert gas at the lungs and tissues," Pharmacol. Rev., vol. 3, pp. 1-41, 1951.

[2] P. Herscovitch, J. Markham, and M. E. Raichle, "Brain blood flow measured with intravenous $\mathrm{H}_{2}^{15} \mathrm{O}$. I. Theory and error analysis," J. Nucl. Med., vol. 24, pp. 782-789, 1983 .

[3] M. E. Raichle, W. R. W. Martin, P. Herscovitch, M. A. Mintun, and J. Markham, "Brain blood flow measured with intravenous $\mathrm{H}_{2}^{15} \mathrm{O}$. II Implementation and validation," J. Nucl. Med., vol. 24, pp. 790-798, 1983.

[4] I. Kanno, A. A. Lammertsma, J. D. Heather, J. M. Gibbs, C. G. Rhodes, J. C. Clark, and T. Jones, "Measurement of cerebral blood flow using bolus inhalation of $\mathrm{C}^{15} \mathrm{O}_{2}$ and positron emission tomography: Description of the method and its comparison with the $\mathrm{C}^{15} \mathrm{O}_{2}$ continuous inhalation method," J. Cereb. Blood Flow Metab., vol. 4, pp. 224-234, 1984.

[5] S.-C. Huang, R. E. Carson, and M. E. Phelps, "Measurement of local cerebral blood flow and distribution volume with short-lived isotopes: A general input technique," J. Cereb. Blood Flow Metab., vol. 2, pp. 99-108, 1982.

[6] R. E. Carson, S.-C. Huang, and M. E. Phelps, "Error analysis of the integrated projection technique and the weighted integration method for measurement of local cerebral blood flow with positron emission tomography," J. Nucl. Med., vol. 25, p. P88, 1984.

[7] R. E. Carson, S.-C. Huang, and M. V. Green, "Weighted integration method for local cerebral blood flow measurements with positron emission tomography," J. Cereb. Blood Flow Metab., vol. 6, pp. 245-258, 1986

[8] N. M. Alpert, L. Eriksson, J. Y. Chang, M. Bergstrom, J. E. Litton, J. A. Correia, C. Bhom, R. H. Ackerman, and J. M. Taveras, "Strategy for the measurement of regional cerebral blood flow using short-lived tracers and emission tomography," J. Cereb. Blood Flow Metab., vol. 4, pp. 28-34, 1984.
[9] G. Blomqvist, "On the construction of functional maps in positron emission tomography," J. Cereb. Blood Flow Metab., vol. 4, pp. 629-632, 1984.

[10] R. A. Koeppe, J. E. Holden, and W. R. Ip, "Performance comparison of parameter estimation techniques for the quantitation of local cerebral blood flow by dynamic positron computed tomography," J. Cereb. Blood Flow Metab., vol. 5, pp. 224-234, 1985.

[11] A. A. Lammertsma, V. J. Cunningham, M. P. Deiber, J. D. Heather, P. M. Bloomfield, J. Nutt, R. S. J. Frackowiak, and T. Jones, "Combination of dynamic and integral methods for generating reproducible functional CBF images," J. Cereb. Blood Flow Metab., vol. 10, pp. 675-686, 1990.

[12] T. Yakoi, I. Kanno, H. Iida, S. Miura, and K. Uemura, "A new approach of weighted integration technique based on accumulated images using dynamic PET and $\mathrm{H}_{2}^{15} \mathrm{O}$," J. Cereb. Blood Flow Metab., vol. 11, pp. 492-501, 1991.

[13] R. E. Carson, "PET parameter estimation using linear integration methods: Bias and variability considerations," in Quantification of Brain Function: Tracer Kinetics and Image Analysis in Brain PET, $\mathrm{K}$. Uemura, N. A. Lassen, T. Jones, and I. Kanno, Eds, Amsterdam, The Netherlands: Excerpta Medica, 1993, pp. 499-507.

[14] D. Feng, Z. Wang, and S.-C. Huang, "A study on statistically reliable and computationally efficient algorithms for generating local cerebral blood flow parametric images with positron emission tomography," IEEE Trans. Med. Imag., vol. 12, pp. 182-188, Mar. 1993.

[15] K. Herholz and C. S. Patlak, "The influence of tissue heterogeneity on results of fitting nonlinear model equations to regional tracer uptake curves: With an application to compartmental models used in positron emission tomography," J. Cereb. Blood Flow Metab., vol. 7, pp. 214-229, 1987.

[16] H. Iida, I. Kanno, S. Miura, M. Murakami, K. Takahashi, and K. Uemura, "A determination of the regional brain/blood partition coefficient of water using dynamic positron emission tomography," J. Cereb. Blood Flow Metab., vol. 9, pp. 874-885, 1989.

[17] K. Schmidt, L. Sokoloff, and S. S. Kety, "Effects of tissue heterogeneity on estimates of regional cerebral blood flow," J. Cereb. Blood Flow Metab., vol. 9, p. S242, 1989. Suppl. 1.

[18] L. Sokoloff, "Local cerebral circulation at rest and during altered cerebral activity induced by anesthesia or visual stimulation," in The Regional Chemistry, Physiology and Pharmacology of the Nervous System, S. S. Kety and J. Elkes, Eds, Oxford, U.K.: Pergamon Press, 1961, pp. 107-117.

[19] O. Sakurada, C. Kennedy, J. Jehle, J. D. Brown, G. L. Carbin, and L. Sokoloff, "Measurement of local cerebral blood flow with iodo $\left[{ }^{14}\right.$ C]antipyrine," Amer. J. Physiol., vol. 234, no. 1, pp. H59-H66, 1978.

[20] S.-C. Huang, R. E. Carson, E. J. Hoffman, J. Carson, N. MacDonald, J. R. Barrio, and M. E. Phelps, "Quantitative measurement of local cerebral blood flow in humans by positron computed tomography and ${ }^{15}$ O-water," J. Cereb. Blood Flow Metab., vol. 3, pp. 141-153, 1983.

[21] P. Herscovitch and M. E. Raichle, "What is the correct value for the brain-blood partition coefficient for water?," J. Cereb. Blood Flow Metab., vol. 5, pp. 65-69, 1985.

[22] H. Iida, I. Kanno, S. Miura, M. Murakami, K. Takahashi, and K. Uemura, "Error analysis of a quantitative cerebral blood flow measurement using $\mathrm{H}_{2}^{15} \mathrm{O}$ autoradiography and positron emission tomography, with respect to the dispersion of the input function," J. Cereb. Blood Flow Metab., vol. 6 , pp. 536-545, 1986.

[23] H. Iida, S. Higano, N. Tomura, F. Shishido, I. Kanno, S. Miura, M. Murakami, K. Takahashi, H. Sasaki, and K. Uemura, "Evaluation of regional differences of tracer appearance time in cerebral tissues using $\left[{ }^{5} \mathrm{O}\right]$ water and dynamic positron emission tomography," J. Cereb. Blood Flow Metab., vol. 8, pp. 285-288, 1988.

[24] J. van den Hoff, W. Burchert, W. Müller-Schauenburg, G.-J. Meyer, and H. Hundeshagen, "Accurate local blood flow measurements with dynamic PET: Fast determination of input function delay and dispersion by multilinear minimization," J. Nucl. Med., vol. 34, pp. 1770-1777, 1993.

[25] T. F. Budinger, S. E. Derenzo, W. L. Greenberg, G. T. Gullberg, and R. H. Huesman, "Quantitative potentials of dynamic emission computed tomography," J. Nucl. Med., vol. 19, pp. 309-315, 1978.

[26] MATLAB Function Reference, Volume 1: Language (1999). [Online]. Available: http://www.mathworks.com/access/helpdesk/help/pdf_doc/matlab/ref/refbook.pdf 
[27] Z. Wang and D. Feng, "Continuous-time system modeling using the weighted-parabola-overlapping numerical integration method," Int. J. Systems Sci., vol. 23, pp. 1361-1369, 1992.

[28] H. Iida, I. Law, B. Pakkenberg, A. Krarup-Hansen, S. Eberl, S. Holm, A. K. Hansen, H. J. G. Gundersen, C. Thomsen, C. Svarer, P. Ring, L. Friberg, and O. B. Paulson, "Quantitation of regional cerebral blood flow corrected for partial volume effect using O-15 water and PET: I. Theory, error analysis, and stereological comparison," J. Cereb. Blood Flow Metab., vol. 20, pp. 1237-1251, 2000.

[29] I. Law, H. Iida, S. Holm, S. Nour, E. Rostrup, C. Svarer, and O. B. Paulson, "Quantitation of regional cerebral blood flow corrected for partial volume effect using O-15 water and PET: II. Normal values and gray matter blood flow response to visual activation," J. Cereb. Blood Flow Metab., vol. 20, pp. 1252-1263, 2000.

[30] S. Pajevic, M. E. Daube-Witherspoon, S. L. Bacharach, and R. E. Carson, "Noise characteristics of 3-D and 2-D PET images," IEEE Trans. Med. Imag., vol. 17, pp. 9-23, Feb. 1998.
[31] H. Herzog, R. J. Seitz, L. Tellmann, E. R. Kops, F. Jülicher, G. Schlaug, A. Kleinschmidt, and H. W. Müller-Gärtner, "Quantitation of regional cerebral blood flow with ${ }^{15} \mathrm{O}$-butanol and positron emission tomography in humans," J. Cereb. Blood Flow Metab., vol. 16, pp. 645-649, 1996.

[32] R. P. Quarles, M. A. Mintun, K. B. Larson, J. Markham, A. M. MacLeod, and M. E. Raichle, "Measurement of regional cerebral blood flow with positron emission tomography: A comparison of $\left[{ }^{15} \mathrm{O}\right]$ water to ${ }^{[11}$ C]butanol with distributed-parameter and compartmental models," J. Cereb. Blood Flow Metab., vol. 13, pp. 723-747, 1993.

[33] J. E. Holden, S. J. Gatley, R. D. Hichwa, W. R. Ip, W. J. Shaughnessy, R. J. Nickles, and R. E. Polcyn, "Cerebral blood flow using PET measurements of fluoromethane kinetics," J. Nucl. Med., vol. 22, pp. 1084-1088, 1981 